\title{
Zooarqueología y análisis de isótopos estables en un contexto pastoril: el caso de Cueva Chayal (Jujuy, Argentina)
}

\section{Zooarchaeology and stable isotope analyses in a pastoralist context: the case study of Chayal Cave (Jujuy, Argentina)}

\author{
Celeste Samec 1,2,3 (iD https://orcid.org/0000-0002-1072-3363 \\ Hugo Yacobaccio ${ }^{4}$ (D) https://orcid.org/0000-0001-6230-4155 \\ ${ }^{1}$ Consejo Nacional de Investigaciones Científicas y Técnicas - Instituto de Geocronología y Geología \\ Isotópica, Universidad de Buenos Aires, Buenos Aires, ARGENTINA. Email: celestesamec@gmail.com \\ ${ }^{2}$ Instituto de Ciencias Naturales Alexander von Humboldt, Universidad de Antofagasta, CHILE. \\ ${ }^{3}$ Archaeology Department, Max Planck Institute for the Science of Human History, Jena, ALEMANIA. \\ ${ }^{4}$ Consejo Nacional de Investigaciones Científicas y Técnicas - Instituto de Arqueología, Facultad de Filosofía \\ y Letras, Universidad de Buenos Aires, ARGENTINA. Email: hdyacobaccio@gmail.com
}

\section{Resumen}

El objetivo de este trabajo consiste en estudiar y discutir las estrategias de explotación de camélidos sudamericanos empleadas por los ocupantes del sitio arqueológico Cueva Chayal (departamento de Susques, provincia de Jujuy, Argentina) durante el tramo final del Holoceno Tardío (entre 680 y 410 años AP). A fin de lograr dicho objetivo se revisará la información zooarqueológica recabada en el sitio y se presentarán valores de isótopos estables $\left(\delta^{13} \mathrm{C}_{\mathrm{VPDB}}\right.$, $\delta^{18} \mathrm{O}_{\mathrm{VPDB}}$ y $\delta^{15} \mathrm{~N}_{\mathrm{AIR}}$ ) medidos sobre hidroxiapatita y colágeno óseo extraídos del material faunístico correspondiente a camélidos domésticos y silvestres. A partir de ello se discutirán las características particulares de las estrategias de pastoreo y caza empleadas por los ocupantes de la cueva y su relación con los cambios ambientales ocurridos en esta área durante el lapso temporal cubierto. En líneas generales, los resultados presentados aquí se muestran consistentes con lo esperado en función de la línea de base construida con datos isotópicos actuales del área, lo que permitió trazar ciertas continuidades en las estrategias de explotación de los camélidos -fundamentalmente el pastoreo- así como en las estrategias de movilidad y uso del espacio en este sector de la Puna Seca entre los siglos XIV y XV de la era.

Palabras clave: pastoreo, caza, camélidos sudamericanos, puna, Holoceno Tardío. 


\begin{abstract}
The aim of this work is to study the South American camelids' exploitation strategies employed by the inhabitants of the archaeological site of Cueva Chayal (Department of Susques, Province of Jujuy, Argentina) during the last part of the Late Holocene (between 680 and 410 years BP). In order to reach this goal, we revise the zooarchaeological information obtained on the site and we present stable isotope values $\left(\delta^{13} \mathrm{C}_{\mathrm{VPDB}}, \delta^{18} \mathrm{O}_{\mathrm{VPDB}}\right.$ y $\left.\delta^{15} \mathrm{~N}_{\mathrm{AIR}}\right)$ measured on bone apatite and bone collagen extracted from the remains of domestic and wild camelids. Based on this, we discuss the characteristics of the herding and hunting strategies employed by the cave inhabitants and their relationship with the environmental changes that took place in this area during the period considered here. Overall, our results are consistent with our expectations based on the isotopic baseline built for the study area using modern isotopic data, an aspect that allow us to propose the existence of certain continuity in the camelids' exploitation strategies -especially pastoralism- as well as the use of the landscape and the mobility strategies employed in this sector of the Dry Puna during the XIV and XV centuries of our Era.
\end{abstract}

Keywords: herding, hunting, South American camelids, Puna, late Holocene.

Recibido: 8 junio 2020. Aceptado: 16 septiembre 2020 


\section{Introducción}

Las estrategias de subsistencia y manejo de recursos practicadas por los grupos humanos en el pasado se han visto condicionadas por las características del medio circundante, entre las que se encuentran el clima o la geomorfología (Schutkowski, 2006). En ambientes áridos y semiáridos, las poblaciones humanas han debido enfrentarse a características desafiantes tales como la escasez de precipitaciones, la sucesión de extensos períodos de sequía y la baja productividad primaria (Laity, 2008). En un escenario como este, el pastoralismo, entendido como la estrategia de subsistencia que consiste en la reproducción y crianza de animales domesticados, puede entenderse como una estrategia eficaz para lidiar tanto con la baja productividad primaria como con la inestabilidad climática, dada su capacidad de transformar vegetación impalatable para los humanos en energía consumible en forma de carne y productos secundarios (Butt, 2010).

Actualmente, el pastoralismo tradicional es practicado por diferentes grupos culturales en diversas áreas de África, Eurasia y Sudamérica (Khazanov, 1994). Estos grupos pastoralistas se organizan a través de una serie de estrategias de subsistencia y movilidad que reflejan las trayectorias particulares de sus ambientes y culturas, las cuales moldean sus prácticas pastoriles en la escala local y regional (Makarewicz, 2013). En las tierras altas de los Andes, el pastoralismo moderno presenta algunas continuidades con las prácticas del pasado en la medida en que se articula en función de la reproducción y crianza de camélidos sudamericanos domesticados, es decir, llamas y/o alpacas (Dransart, 2011).

La evidencia arqueológica recuperada en las tierras altas de los Andes sugiere que la domesticación de los camélidos sudamericanos tuvo lugar entre los 6000 y los 4000 años AP (Moore, 2016), posibilitando el acceso regular a diversos productos animales (carne, fibra, guano) así como también el desarrollo de redes de intercambio de larga distancia a través de las caravanas de llamas (Mengoni Gońalons y Yacobaccio, 2006). Posteriormente, ya durante el Holoceno Tardío, el pastoreo de camélidos domesticados se afianza como estrategia económica en gran parte de la región andina, adquiriendo características propias en cada sector (Capriles y Tripcevich, 2016). Particularmente, en las tierras altas de la puna argentina se producen diversos cambios en las estrategias de subsistencia y explotación de las poblaciones de camélidos sudamericanos hacia fines de dicho período, ligadas fundamentalmente al establecimiento del pastoreo como estrategia predominante (Yacobaccio, Madero, Malmierca y Reigadas, 19971998). En el extremo sudoccidental de la Puna Seca, se establece el desarrollo de un primer momento de "Pastoreo-Caza" entre los 1100 y los 550 ańos AP, lapso en el cual el pastoreo de llamas se constituye como la actividad fundamental, tal como lo muestran las evidencias recabadas en sitios como Cueva Quispe, Puesto Demetria o Cueva Chayal (Yacobaccio et al., 2011). Es para este momento cronológico que se postula la existencia de un patrón de asentamiento fuertemente determinado por las actividades pastoriles, que se asemeja al modo en el que las comunidades que habitan esta área utilizan el ambiente actualmente (Yacobaccio et al., 1997-1998). Este patrón de asentamiento era integrado por sitios relativamente grandes (alrededor de $5000 \mathrm{~m}^{2}$ ) ubicados cerca de vegas o vertientes y sitios pequeńos (solo decenas de $\mathrm{m}^{2}$ ) ubicados en fondos de quebradas. Los primeros estaban compuestos por varios recintos (más de cuatro y en algunos casos hasta 13), mientras que los segundos incluían una o dos estructuras de piedra (Yacobaccio et al., 1997-1998). Por otra parte, existe un segundo momento de "Pastoreo-Caza" que se sitúa entre los 550 y los 220 ańos AP, lapso en el cual estas mismas actividades de subsistencia se ven complementadas por un acceso temporal a cultivos 
de otras áreas, principalmente maíz, y la aparición de fauna exótica (Caprinae, Bovinae), tal como evidencia el registro de sitios como Puesto Demetria, Chulpa Chayal y Lapao Chico 1 y 2 en el departamento jujeño de Susques (Yacobaccio et al., 2011). Es importante destacar que aún en estos momentos en los que la actividad determinante es el pastoreo, la caza no perdió relevancia y habría cumplido un papel importante en mantener la viabilidad reproductiva de los rebaños, actuando también como un reaseguro frente a cualquier eventualidad climática (Olivera y Grant, 2009). Al mismo tiempo, vale mencionar que durante el transcurso de estos dos períodos de "Pastoreo-Caza" el área de estudio presenta condiciones ambientales variables, ligadas a eventos tales como la Anomalía Climática Medieval y la Pequeña Edad de Hielo, que condujeron a una creciente fragmentación ambiental (Lupo, Morales, Yacobaccio, Maldonado y Grosjean, 2007; Morales et al., 2009; Morales et al., 2012).

Considerando todo lo expuesto anteriormente, el objetivo de este trabajo consiste en explorar las estrategias de movilidad, uso del espacio y explotación de los camélidos sudamericanos empleadas por los grupos humanos que ocuparon la Puna Seca de Argentina a partir del estudio de los materiales recuperados en el sitio Cueva Chayal (departamento de Susques, provincia de Jujuy). A fin de lograr esto se integrarán los resultados del análisis zooarqueológico y de isótopos estables $\left(\delta^{13} \mathrm{C}_{\mathrm{VPDB}}, \delta^{15} \mathrm{~N}_{\mathrm{AIR}}, \delta^{18} \mathrm{O}_{\mathrm{VPDB}}\right)$ efectuado sobre las arqueofaunas recuperadas en la capa 3 del sitio, la cual cuenta con fechados entre los 680 y los 410 años AP (ca. 1296 y 1510 años de la EC). A partir de ello se discutirá el desarrollo del pastoreo de camélidos domésticos -registrado en el área de estudio desde al menos 2600 ańos AP-y su asociación con la caza de camélidos silvestres, seguido de la posterior estabilización de un sistema pastoril-cazador que se mantendrá hasta momentos históricos (Yacobaccio et al., 1997-1998).

\section{Antecedentes relevantes para el desarrollo de este trabajo}

Los camélidos sudamericanos se perfilaron como un recurso crítico desde las primeras ocupaciones humanas en la región andina, sustentando en tiempos tardíos la única estrategia de domesticación de mamíferos de gran tamaño de las Américas (Moore, 2016). En la región andina en general y en la puna de Atacama en particular se han llevado a cabo investigaciones que analizan las relaciones que los grupos humanos establecieron con las poblaciones de camélidos para distintos períodos y sectores desde numerosas líneas de evidencia (Dransart, 1991; Olivera, 1997; Elkin y Rosenfeld 2001; López, 2003; Gallardo y Yacobaccio, 2005; Cartajena, Núñez y Grosjean, 2007, por mencionar algunos). Una de estas líneas de evidencia viene representada por los estudios zooarqueológicos, que a través del análisis de las características taxonómicas, morfológicas y osteométricas de los elementos que forman parte de los conjuntos faunísticos, permiten evaluar la presencia de animales domésticos y/o silvestres y las estrategias de explotación de los mismos implementadas por los grupos humanos en diferentes momentos cronológicos (Olivera y Elkin, 1994; Mengoni Goñalons y Yacobaccio, 2006; Cartajena, 2013, entre muchos otros). Recientemente, la información otorgada por los estudios zooarqueológicos se ha visto complementada por aquella generada a partir de los análisis de isótopos estables. De tal manera, en las últimas décadas diversos estudios han analizado la composición isotópica del material óseo de camélidos sudamericanos provenientes de diversas áreas de los Andes centrales y regiones adyacentes, con el objetivo de estudiar las estrategias de explotación y manejo de estos animales en diversos contextos (Finucane, Maita Agurto e Isbell, 2006; Knudson, Gardella y Yaeger, 2012; López, Cartajena y Núñez, 2013; Dufour et al., 2014; Szpak, Millaire, White y Longstaffe, 2014; López, Cartajena, Loyola, Núñez y Carrasco, 2017; Mondini et al., 2019, entre otros). En el Noroeste Argentino (NOA), el análisis isotópico de tejidos de camélidos ha permitido explorar diversas problemáticas tales como la explotación de camélidos 
silvestres durante la primera parte del Holoceno en la puna de Catamarca (Mondini, Panarello y Zangrando, 2010; Mondini y Panarello 2014), las estrategias de manejo de rebańos durante el primer milenio de la era en diferentes contextos arqueológicos del área valliserrana de Salta y Catamarca (Izeta, Laguens, Marconetto y Scattolin, 2009; Dantas, Figueroa y Laguens, 2014) y las estrategias de caza y pastoreo en contextos incaicos de valles y quebradas de Salta y Jujuy (Mengoni Goñalons, 2007, 2014), solo por enumerar algunos de los casos más relevantes. Vale mencionar que hasta el momento muchos de los trabajos que presentan valores de isótopos estables para camélidos en el NOA solo analizan la composición isotópica del carbono (Izeta et al., 2009; Dantas, 2012; Grant y Olivera 2016) aunque algunas investigaciones han incluido en la discusión la composición isotópica del nitrógeno (Srur, Izeta y Scattolin, 2012; Mengoni Gońalons, 2014; Grant, Mondini y Panarello, 2018) y el estroncio (Dantas y Knudson, 2016). Por otra parte, hasta el momento existe un único antecedente de análisis de isótopos estables del oxígeno para el estudio de las poblaciones de camélidos en el NOA, el cual identificó la existencia de una correlación entre la composición isotópica del agua meteórica y la sangre de camélidos actuales silvestres y domesticados (Fernández y Panarello, 1989-1990). En este sentido, vale mencionar que parte de los antecedentes con los que contamos para el área de estudio se han dedicado a establecer líneas de base para interpretar datos provenientes de sitios arqueológicos, midiendo la composición isotópica del carbono y el nitrógeno en plantas (Fernández y Panarello, 1999-2001; Samec, Yacobaccio y Panarello, 2017) y la composición isotópica del oxígeno de aguas meteóricas y superficiales (Fernández y Panarello, 1989-1990; Samec, Morales, Pirola, Panarello y Yacobaccio, 2017). Estos antecedentes demuestran que la distribución de las plantas $\mathrm{C}_{3} \mathrm{y} \mathrm{C}_{4}$ se encuentra correlacionada con la variable altitudinal, de acuerdo con sus efectos en la temperatura y la precipitación (Samec, Yacobaccio, et al., 2017). De tal forma, si bien las plantas $\mathrm{C}_{3}$ son las más abundantes en todas las comunidades vegetales muestreadas, las plantas $\mathrm{C}_{4}$ se encuentran presentes en áreas de menor altitud debido a condiciones de mayor temperatura y estrés hídrico, aunque prácticamente desaparecen por encima de los $3900 \mathrm{msnm}$ donde estas condiciones ya no se presentan (Samec, Yacobaccio, et al., 2017). Por otro lado, se han observado valores de $\delta^{15} \mathrm{~N}_{\mathrm{AIR}}$ más elevados en aquellas plantas que se encuentran en ambientes áridos y salinos, mientras que los valores más bajos se encuentran en ambientes más húmedos que cuentan con mayor precipitación (Samec, Yacobaccio, et al., 2017). Estos patrones se reflejan a su vez en los valores de $\delta^{13} \mathrm{C}_{\mathrm{VPDB}}$ y $\delta^{15} \mathrm{~N}_{\mathrm{AIR}}$ medidos sobre colágeno óseo de camélidos (silvestres y domésticos) modernos, los cuales presentan una correlación negativa con la altitud en ambos casos (Yacobaccio, Morales y Samec, 2009; Samec, Yacobaccio y Panarello, 2018). En el caso de los valores de $\delta^{18} \mathrm{O}_{\text {VPDB }}$ medidos sobre carbonatos o fosfatos de la hidroxiapatita ósea, resulta importante considerar la variabilidad de las posibles fuentes de agua consumida por los camélidos y su variabilidad en términos de composición isotópica, la cual viene determinada por diversos factores tales como la naturaleza de la fuente de agua y su localización, la incidencia de las precipitaciones y la temperatura ambiente (Knudson, 2009). Los estudios realizados hasta el momento han evidenciado un patrón de lluvias más empobrecidas a medida que se incrementa la altitud (Fernández y Panarello, 1989-1990) en consonancia con lo planteado por Dansgaard (1964), así como una gran variabilidad en los valores de las aguas superficiales recolectados en una temporada, notablemente afectados en muchos casos por efecto de la evaporación (Samec, Morales, et al., 2017).

Considerando los antecedentes expuestos más arriba, este trabajo apunta a integrar las evidencias generadas a partir del análisis zooarqueológico realizado sobre el conjunto arqueofaunístico del sitio Cueva Chayal con los resultados de los análisis de la composición isotópica del carbono, el nitrógeno y el oxígeno sobre los materiales óseos asignados a camélidos domésticos y silvestres recuperados en el sitio. Si bien una fracción de los resultados presentados aquí ha 
sido parte de una publicación previa (Samec, Pirola, Yacobaccio y Panarello, 2020), en esta ocasión se suman los valores de $\delta^{13} \mathrm{C}_{\mathrm{VPDB}}$ y $\delta^{15} \mathrm{~N}_{\mathrm{AIR}}$ medidos sobre colágeno óseo de huesos asignados a la especie silvestre Vicugna vicugna, así como también los valores de $\delta^{13} \mathrm{C}_{\mathrm{VPDB}} \mathrm{y}$ $\delta^{18} \mathrm{O}_{\mathrm{VPDB}}$ medidos sobre hidroxiapatita ósea de especímenes asignados a Vicugna vicugna y Lama glama. El objetivo final de este artículo entonces es el de integrar toda la información con la que contamos para el sitio en cuestión y así lograr una discusión más profunda de las estrategias de explotación de los camélidos domésticos y silvestres para momentos tardíos en la Puna Seca. En este sentido, consideramos que integrar los datos que tenemos en el estudio conjunto de las estrategias de pastoreo y caza nos permitirá estudiar en detalle la articulación de las estrategias de subsistencia y su relación con las prácticas de movilidad y uso del paisaje por parte de los grupos humanos que ocuparon el sitio.

\section{El área de estudio}

La puna argentina comprende las tierras altas de este país situadas entre los 22 grados y los 27 grados de latitud Sur sobre la cordillera de los Andes, con una altitud que varía entre los 3000 y los 5000 msnm (Figura 1). Esta región es considerada como un desierto de altura que comprende distintas comunidades vegetales cuya distribución se ve determinada por la altitud (Cabrera, 1976). Algunas de sus características más importantes son una amplia variación térmica entre el día y la noche, una alta radiación solar y una baja presión atmosférica debido a la altitud. Los recursos acuíferos se distribuyen de manera irregular en el espacio, existiendo una red hidrográfica pobremente desarrollada, de forma que las únicas fuentes de agua dulce son unos pocos ríos y algunos manantiales dispersos. La productividad primaria se encuentra concentrada en sectores específicos del paisaje, a menudo vinculados a fuentes estables de humedad tales como cuencas primarias, quebradas altas o vegas. Las lluvias ocurren durante el verano y se encuentran determinadas por el sistema monsónico de América del Sur (Zhou y Lau, 1998). Al mismo tiempo, las precipitaciones exhiben un gradiente latitudinal, la cual delimita dos subáreas dentro de la puna argentina: la Puna Seca, localizada al norte de los $24^{\circ}$ $\mathrm{S}$, con una precipitación media anual de $300 \mathrm{~mm} / \mathrm{ańo}$, y la Puna Salada, localizada al sur de los $24^{\circ} \mathrm{S}$, con una precipitación media anual que apenas alcanza los $100 \mathrm{~mm} / \mathrm{año}$ (Bianchi, Yañez y Acuña, 2005).

En este trabajo, nos enfocaremos en un área específica dentro de la denominada Puna Seca, la cual corresponde a las inmediaciones del poblado de Susques, en el departamento homónimo dentro de la provincia de Jujuy (ver Figura 1). El área de Susques está localizada en una terraza aluvial a $3600 \mathrm{msnm}$ cerca de la confluencia de los ríos Susques y Pastos Chicos en la transición entre la Puna Seca y la Salada. Su morfología está conformada por un plateau de tobas ignimbríticas con una altura media de $3900 \mathrm{msnm}$, disectado por profundas quebradas y enmarcado por la sierra de Taire al oeste y la de Cobres al este. En este sector el clima es frío y seco, con precipitaciones estivales (diciembre-marzo) que promedian los $190 \mathrm{~mm}$ anuales. La temperatura media es de $7,5^{\circ} \mathrm{C}$ y se registran heladas durante todo el ańo. Es importante destacar que en este sector la variación anual en la cantidad y recurrencia de las precipitaciones es muy alta y puede alcanzar hasta un 60\%. Las sequías son frecuentes y en muchos casos duraderas; al menos uno de cada cuatro años registra una sequía severa durante la cual llueve entre un 40 y un $45 \%$ por debajo del promedio general. 


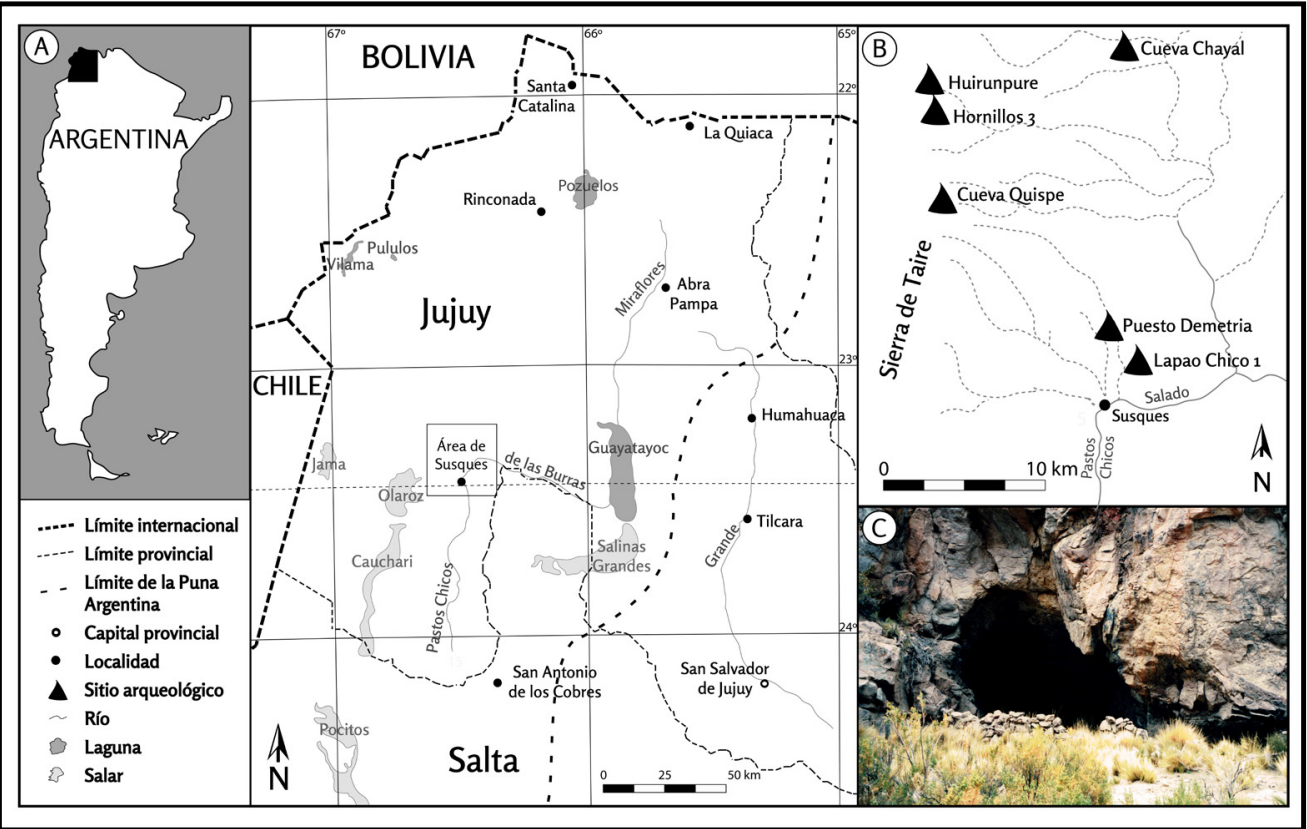

Figura 1. Mapa del área de estudio.

A) Localización del área de Susques dentro de la Puna Argentina; B) Localización de la Cueva Chayal y otros sitios mencionados en el texto dentro del área de Susques; C) Imagen de la Cueva Chayal.

\section{El sitio de Cueva Chayal}

La Cueva Chayal está localizada en la quebrada de Chayal dentro del departamento de Susques (provincia de Jujuy, República Argentina), a unos $3 \mathrm{~km}$ del pequeño poblado de San Juan de Quillaques y a una altitud de $3700 \mathrm{msnm}$. Vale mencionar que la quebrada de Chayal representa un "microambiente" debido a la presencia de recursos acuíferos y la alta concentración de pasturas (p.e., Stipa sp., Festuca sp.). La cueva cuenta con $20 \mathrm{~m}^{2}$ de superficie, de los cuales se excavaron $4,5 \mathrm{~m}^{2}$, ya que no toda su superficie es excavable, permitiendo detectar cinco capas naturales que contenían tres niveles de ocupación (capas 2, 3 y 4 ) (Yacobaccio et al., 1997-1998). En las excavaciones se recuperó gran cantidad de restos óseos bien conservados, una buena cantidad de fragmentos cerámicos y escaso material lítico. De los dos fechados efectuados en materiales recuperados en la capa 3 del sitio, el más tardío proviene de un fogón en cubeta rodeado de lajas, mientras que el más temprano, de carbones sueltos recuperados en la capa (Tabla 1).

La cueva está asociada a un sitio de mayor tamaño denominado Chayal 2 y compuesto por cinco recintos de piedra con muros perimetrales en los sectores este y oeste, mientras que los bordes norte-sur están delimitados por bardas de ignimbrita. Sobre un resalte de las mismas se hallan siete trojas o silos de almacenamiento con una capacidad de $30,5 \mathrm{~m}^{3}$. Aunque la mayoría de los recintos están erosionados y presentan piso de roca; en uno de ellos se pudo practicar un sondeo y se obtuvo una datación de 610 50 ańos AP o cal DC 1298-1442 (2б) (UGA-8720, carbón) a partir del fechado de carbones sueltos extraídos del mismo. Cabe destacar que esta fecha no presenta diferencias estadísticas con las obtenidas de la capa 3 de Cueva Chayal, aspecto que parece apuntar a la contemporaneidad de ambas ocupaciones. Si tomamos en cuenta 
Tabla 1. Fechados radiocarbónicos de la Cueva Chayal (capas 3 y 4).

\begin{tabular}{|l|c|c|c|c|}
\hline Código muestra & ${ }^{14}$ C ańos AP & Cal 2 o DC & Material & $\mathbf{N}^{\circ}$ de laboratorio \\
\hline Cueva Chayal c.4 & $1080 \pm 80$ & $774-1205$ & Carbón & LP 375 \\
\hline Cueva Chayal c.3 & $680 \pm 50$ & $1282-1403$ & Carbón & LP 325 \\
\hline Cueva Chayal c.3 & $410 \pm 50$ & $1448-1632$ & Carbón & LP 398 \\
\hline
\end{tabular}

Las fechas calibradas fueron calculadas utilizando la curva SHCal20 en el programa Calib 8.1.0 (Stuiver y Reimer, 1993; Hogg et al., 2020).

la datación del fogón de dicha capa 3 que representaría el fin de la ocupación, podríamos estimar el rango temporal al que nos estamos refiriendo aquí entre 1282 y 1632 cal DC tomando en cuenta dos sigmas; pero si ajustamos la calibración conjunta de las tres fechas estaríamos considerando probable un rango de ocupación de los dos sitios entre 1296 y 1510 cal DC. En este sentido, consideramos que la Cueva Chayal probablemente haya funcionado como un área de actividades específicas (¿cocina quizás?) vinculada al sitio Chayal 2 (Samec et al., 2020).

Entre los materiales arqueológicos recuperados en la localidad de Chayal, podemos mencionar los 170 fragmentos de cerámica obtenidos en Chayal 2, los cuales corresponden mayormente a vasijas de tamańo grande y paredes gruesas (Solá, 2007). Asimismo, dentro de la capa 3 de la Cueva Chayal se recuperaron 183 fragmentos que incluyen piezas grandes (ollas), pero también vasijas más pequeñas y platos. Ambos sitios arrojaron tiestos provenientes de otras áreas: en el caso de la Cueva Chayal se encontró un tipo Poma negro sobre rojo y en Chayal 2 se recuperaron 14 fragmentos importados de otras regiones de la puna argentina y la quebrada de Humahuaca (p.e., tipos "Yavi", cerámicas con puntos blancos y reticulados negro sobre rojo) (Solá, 2011). Asimismo, es relevante destacar que de la capa 3 de Cueva Chayal se recuperó una azada de pizarra completa.

\section{El conjunto faunístico de Cueva Chayal}

En cuanto a los materiales arqueofaunísiticos, la capa 3 del sitio Cueva Chayal reúne el $84 \%$ del total de especímenes óseos recuperados en el sitio $(n=1291)$ y representa el material analizado en este trabajo. Dentro de las especies presentes se destacan en primer lugar las vizcachas (Lagidium sp.) y en segundo lugar los camélidos, en aparente relación con la disponibilidad local del primer taxón en los roqueríos que rodean al sitio (Tabla 2) (Yacobaccio et al., 1997-1998).

A fin de establecer la integridad del subconjunto correspondiente a la familia Camelidae, se analizó la relación existente entre la densidad ósea y la abundancia de huesos identificados a partir del cálculo del coeficiente de correlación por rangos (rho de Spearman) entre los valores de densidad global establecidos para llama (Elkin, 1995) y los valores de especímenes óseos identificados (NISP) (Tabla 3). Los resultados obtenidos indican que las partes esqueletarias 
Tabla 2. Abundancia taxonómica de las arqueofaunas recuperadas en la capa 3 del sitio Cueva Chayal.

\begin{tabular}{|l|c|c|c|c|}
\hline Taxón & NISP & \% & MNI & \% \\
\hline Camelidae & 175 & 38 & 12 & \\
\hline Artiodactyla & 49 & 10 & 11 & 47 \\
\hline Lagidium sp. & 230 & 50 & & \\
\hline Roedor pequeño & 5 & 1 & & \\
\hline Total & 459 & 99 & 23 & 99 \\
\hline
\end{tabular}

presentes en la capa 3 de Cueva Chayal no exhiben una correlación significativa con la densidad ósea $\left(r_{s}=0,12 p<0,05\right)$, revelando una baja incidencia de esta en la composición del conjunto analizado aquí. Al mismo tiempo, solo el $4 \%$ de las partes esqueletarias asignadas a artiodáctilos y camélidos presenta signos de meteorización en estadios superiores a 2 según la escala de Behrensmeyer (1978). Por otra parte, vale mencionar que los materiales analizados aquí no presentan indicios de haber sido expuestos al fuego de manera directa, al tiempo que no se recuperaron especímenes óseos en el interior del fogón excavado. Esta información permite descartar el asado como método de cocción empleado en el sitio, dando lugar a la suposición de que parte del conjunto fuera cocido a partir del hervido de partes en contenedores de cerámica, en consonancia con el grado y tipo de fragmentación que presentan muchos de los huesos asignados a artiodáctilos y camélidos (Yacobaccio et al., 1997-1998).

Ahora bien, dentro de los especímenes asignados a camélidos, predomina el esqueleto apendicular sobre el axial y se destacan principalmente los huesos de la parte inferior de las patas, siendo el pie trasero y las falanges las partes esqueletarias que resultan más abundantes. La preponderancia de estas partes dentro del conjunto considerado aquí coincide con una estrategia que habría privilegiado a la categoría 3 de las llamadas Partes Esqueletarias Básicas (PEB) que incluye fundamentalmente los huesos de las extremidades -es decir, radioulna, metatarso, metacarpo, falanges-, los cuales aportan un alto contenido de médula y un bajo contenido de carne. Las PEB permiten evaluar los mecanismos de selección de las unidades anatómicas en función de su rendimiento económico y se calculan sumando las proporciones de cada NME (Número Mínimo de Elementos) y agrupándolas según zonas del esqueleto (cabeza, columna, costillas, etc.) que coinciden con las unidades de trozamiento empleadas por los pastores actuales luego del desposte inicial (Yacobaccio et al., 1997-1998) (Tabla 3). En este caso, la preponderancia de partes correspondientes a la categoría 3 marca una estrategia destinada al aprovechamiento de la grasa contenida en la médula ósea en oposición a otras categorías (1 y 2) que permiten obtener un mayor porcentaje de carne (Yacobaccio et al., 1997-1998). Asimismo, vale mencionar que el perfil esqueletario de los camélidos recuperados en el sitio estaría de acuerdo con su uso como base residencial, según los modelos elaborados a partir de estudios etnoarqueológicos realizados en el área de estudio (Yacobaccio et al., 1997-1998). 
Tabla 3. Partes esqueletarias expresadas en NISP y en MNE.

\begin{tabular}{|c|c|c|}
\hline Parte esqueletaria & NISP & MNE \\
\hline Cráneo & 8 & 2 \\
\hline Maxilar & 1 & 1 \\
\hline Mandíbula & 10 & 5 \\
\hline Axis & 2 & 2 \\
\hline Vértebras cervicales & 2 & 2 \\
\hline Vértebras torácicas & 1 & 1 \\
\hline Vértebras lumbares & 2 & 2 \\
\hline Sacro & 1 & 1 \\
\hline Esternebra & 1 & 1 \\
\hline Costillas & 18 & 8 \\
\hline Escápula & 5 & 2 \\
\hline Húmero & 11 & 5 \\
\hline Radioulna & 18 & 6 \\
\hline Carpo & 1 & 1 \\
\hline Metacarpo & 3 & 2 \\
\hline Pelvis & 9 & 4 \\
\hline Fémur & 9 & 5 \\
\hline Tibia & 17 & 5 \\
\hline Astrágalo & 3 & 2 \\
\hline Calcáneo & 3 & 2 \\
\hline Tarso & 4 & 4 \\
\hline Metatarso & 2 & 2 \\
\hline Sesamoideos & 1 & 1 \\
\hline Metapodio & 27 & 7 \\
\hline Falange 1 & 9 & 5 \\
\hline Falange 2 & 4 & 4 \\
\hline Falange 3 & 3 & 3 \\
\hline Totales & 175 & 85 \\
\hline
\end{tabular}

En cuanto a la edad de los camélidos explotados, un $45 \%$ ha sido asignado a individuos adultos, mientras que un $33 \%$ corresponde a nonatos/neonatos y un $21 \%$ a juveniles/inmaduros según los estadios de fusión de las epífisis de los huesos largos (Kent, 1982). La información de erupción y desgaste dentario confirma las proporciones citadas, dado que a partir de cuatro especímenes mandibulares y maxilares se ha determinado que dos corresponden a animales de entre 11 y 13 ańos, uno a un individuo menor a seis meses y el otro a un nonato/neonato 
(Yacobaccio et al., 1997-1998). Esta estructura de edad es marcadamente diferente a la resultante del pastoreo actual, aspecto que podría deberse a la importancia diferencial de la caza en la economía de los ocupantes del sitio (Yacobaccio et al., 1997-1998). En cuanto a las especies de camélidos presentes, dentro de los especímenes completos que pudieron ser medidos se encuentran metacarpos que fueron indiscutidamente asignados a llama (Yacobaccio et al., 1997-1998). También vale la pena mencionar que dentro de los restos óseos asignados a camélidos cerca de un $8 \%$ de los mismos presenta huellas de carnívoros, mientras que un $4 \%$ exhibe huellas de roedores.

\section{Materiales y métodos}

\section{Criterios de selección de las muestras}

Al examinar el conjunto arqueofaunístico recuperado en la capa 3 del sitio Cueva Chayal con el objetivo de seleccionar los especímenes de Camelidae para realizar los análisis isotópicos, se tuvieron en cuenta distintos criterios metodológicos. En primera instancia se prestó especial atención a la procedencia (capa y cuadrícula) del material, además de su asignación esqueletaria, lateralidad y estado de fusión de cada espécimen a fin de evitar la repetición de mediciones sobre el mismo individuo. Al mismo tiempo y siempre que fuera posible, se privilegió la selección de animales maduros cuya edad de muerte fuera estimada en función del estadio de fusión de las epífisis de los huesos largos (Kent, 1982). Por otra parte, en todos los casos se seleccionaron huesos que presentaran buen aspecto visual, con estadios bajos de meteorización (por debajo del estadio 3 de Behrensmeyer 1978) y que no exhibieran señales de termoalteración de ningún tipo. $\mathrm{Y}$ en última instancia, se tomaron muestras de los tejidos densos de las piezas seleccionadas, preferentemente diáfisis, evitando la utilización del tejido esponjoso debido a la mayor posibilidad de contaminación a partir del entrampamiento de sedimento u otras partículas ajenas al tejido analizado.

\section{Protocolos de laboratorio}

La extracción de colágeno óseo de los materiales seleccionados se llevó a cabo en el Instituto de Geocronología y Geología Isotópica (Ciudad Universitaria, Buenos Aires, Argentina) y siguió el protocolo sugerido por Tykot (2004) con algunas modificaciones. Primeramente, se tomó un gramo de tejido óseo compacto por espécimen seleccionado según los criterios enumerados previamente. Esta fracción de hueso fue limpiada físicamente con un torno Dremel ${ }^{\circledR}$ y luego lavada en agua deionizada durante 45 minutos mediante el uso de una lavadora ultrasónica a fin de remover partículas contaminantes. Luego, se llevó a cabo la demineralización sumergiendo el fragmento óseo en ácido clorhídrico al 2\% durante 72 horas. Al mismo tiempo, de manera previa y posterior a la demineralización se realizó la eliminación de ácidos húmicos con hidróxido de sodio con una dilución de $0,1 \mathrm{M}$ durante 24 horas cada vez. Entre paso y paso siempre se procedió a enjuagar la muestra tres veces en agua desionizada antes de proceder al paso siguiente. Para finalizar el pretratamiento las muestras fueron secadas en un horno a $<70$ ${ }^{\circ} \mathrm{C}$ de temperatura.

Por otra parte, la extracción del carbonato de la hidroxiapatita ósea se realizó en el Laboratorio de Isótopos Estables en Ciencias Ambientales (San Rafael, Mendoza, Argentina) y siguió el protocolo empleado por Crowley y Wheatley (2014) con algunas modificaciones. Inicialmente, se limpió la superficie del hueso con una herramienta Dremel $^{\circledR}$ para luego pulverizar 
aproximadamente $30 \mathrm{mg}$ de hueso. Este material luego fue tratado con peróxido de hidrógeno al 3\% durante 15 minutos a fin de eliminar la fracción orgánica del hueso. Luego, las muestras fueron enjuagadas en agua destilada, renovando el agua en tres oportunidades y empleando una centrífuga en cada oportunidad para minimizar la pérdida de muestra con cada enjuague. Posteriormente, las muestras fueron tratadas con $0,1 \mathrm{M}$ de ácido acético durante 15 minutos, seguido de un enjuague de las mismas características que el anterior. Finalmente, las muestras fueron secadas en un horno a $<70{ }^{\circ} \mathrm{C}$ de temperatura.

\section{Espectrometría de masas}

La medición de los valores de $\delta^{13} \mathrm{C}_{\mathrm{VPDB}}$ y $\delta^{15} \mathrm{~N}_{\mathrm{AIR}}$ sobre las muestras de colágeno óseo se realizó en el Instituto de Geocronología y Geología Isotópica (INGEIS) y utilizó un analizador elemental CarloErba (CHONS) acoplado a un espectrómetro de masas de flujo continuo Finnigan MAT Delta V (CF-IRMS) a través de una interfaz Thermo ConFlo IV. Dichas mediciones se realizaron a partir del uso de materiales de referencia internos al laboratorio, calibrados según los estándares internacionales (VPDB para carbono y AIR para nitrógeno) con un error analítico de $\pm 0,2 \%$ p para los valores de $\delta^{13} \mathrm{C}_{\mathrm{VPDB}}$ y $\delta^{15} \mathrm{~N}_{\mathrm{AIR}}$.

Por otra parte, la medición de la composición isotópica del carbono y el oxígeno sobre la fracción carbonato de la hidroxiapatita se realizó en la Stable Isotope Facility de la Universidad de Wyoming (UW SIF) empleando un Thermo Finnigan GasBench II acoplado a un espectrómetro de masas Thermo Finnigan Delta Plus XP (GC-IRMS). El proceso de espectrometría de masas contempló la medición del gas $\mathrm{CO}_{2}$ generado a partir de la reacción del carbonato de la muestra con ácido fosfórico al 100\%. Las mediciones se realizaron a partir del uso de materiales de referencia internos al laboratorio, calibrados según los estándares internacionales (VPDB para carbono y oxígeno). En este caso, el error analítico fue de $\pm 0,1 \%$ o para los valores de $\delta^{13} \mathrm{C}_{\mathrm{VPDB}}$ y de $\pm 0,2 \%$ o para los valores $\delta^{18} \mathrm{O}_{\mathrm{VPDB}}$.

\section{Resultados}

Se analizó la composición isotópica del carbono y el nitrógeno del colágeno extraído de 12 especímenes óseos asignados a camélidos domésticos y silvestres a partir de su tamaño, al tiempo que para nueve de estos especímenes también se analizó la composición del carbono y el oxígeno de la hidroxiapatita. De los valores de $\delta^{13} \mathrm{C}_{\mathrm{VPDB}}$ y $\delta^{15} \mathrm{~N}_{\mathrm{AIR}}$ medidos sobre colágeno se considera aquí la totalidad de los valores obtenidos debido a que en este caso los resultados del cálculo de la relación C:N para cada muestra analizada se encuentran dentro del rango esperado -es decir, entre 2,9 y 3,6- (DeNiro, 1985), permitiendo descartar contaminaciones y alteraciones posdepositacionales (ver Tabla 3). Desgraciadamente, en el caso de los valores $\delta^{13} \mathrm{C}_{\mathrm{VPDB}}$ y $\delta^{18} \mathrm{O}_{\mathrm{VPDB}}$ medidos sobre el carbonato de la hidroxiapatita, no contamos con los recursos necesarios para realizar análisis complementarios (FTIR o DRX) que atestigüen el carácter primario de la señal isotópica, aunque consideramos que la buena preservación del colágeno y la coherencia relativa de los resultados presentados aquí constituyen indicadores de la buena conservación general de los materiales óseos recuperados en el sitio. 
Tabla 4. Valores de $\delta^{13} \mathrm{C}_{\mathrm{VPDB}}$ y $\delta^{15} \mathrm{~N}_{\mathrm{AIR}}$ medidos sobre colágeno óseo y valores de $\delta^{13} \mathrm{C}_{\mathrm{VPDB}} \mathrm{y}$ $\delta^{18} \mathrm{O}_{\mathrm{VPDB}}$ medidos sobre la fracción carbonato de la hidroxiapatita ósea de los materiales seleccionados de la capa 3 del sitio Cueva Chayal.

\begin{tabular}{|c|c|c|c|c|c|c|c|c|}
\hline 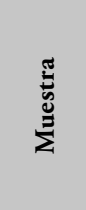 & 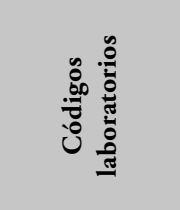 & 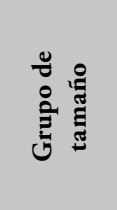 & 茪 & $\frac{\stackrel{8}{0}}{0_{0}^{\infty}}$ & $\frac{2}{0}$ & ت̆ & 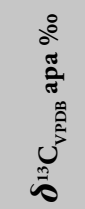 & 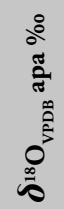 \\
\hline $\mathrm{CC} 1$ & $\begin{array}{c}\text { AIE } 25819 \\
\text { - SIF } \\
20170090.014\end{array}$ & pequeño & falange & $-18,2$ & $+8,4$ & 3,2 & $-10,2$ & $-4,4$ \\
\hline CC11 & $\begin{array}{c}\text { AIE } 25820 \\
- \text { SIF } \\
20170090.022\end{array}$ & pequeño & radio & $-17,1$ & $+9,0$ & 3,2 & $-9,1$ & $-1,9$ \\
\hline CC3 & $\begin{array}{c}\text { AIE } 25821 \\
\text { - SIF } \\
20170090.016\end{array}$ & pequeño & metacarpo & $-18,3$ & $+5,4$ & 3,2 & $-10,6$ & $-3,3$ \\
\hline CC5 & $\begin{array}{c}\text { AIE } 25822 \\
- \text { SIF } \\
20170090.018\end{array}$ & pequeño & metatarso & $-17,9$ & $+5,4$ & 3,3 & $-9,1$ & $-3,7$ \\
\hline CC4 & $\begin{array}{c}\text { AIE } 25826 \\
- \text { SIF } \\
20170090.017\end{array}$ & grande & metapodio & $-18,3$ & $+8,2$ & 3,4 & $-10,6$ & $-2,6$ \\
\hline CC6 & $\begin{array}{c}\text { AIE } 25827 \\
\text { - SIF } \\
20170090.019\end{array}$ & grande & fémur & $-17,2$ & $+8,6$ & 3,3 & $-10,1$ & $-1,7$ \\
\hline CC7 & $\begin{array}{c}\text { AIE } 25828 \\
- \text { SIF } \\
20170090.020\end{array}$ & grande & falange & $-18,4$ & $+6,6$ & 3,3 & $-9,5$ & $-4,3$ \\
\hline CC9 & $\begin{array}{c}\text { AIE } 25829 \\
\text { - SIF } \\
20170090.021\end{array}$ & grande & fémur & $-17,3$ & $+7,5$ & 3,3 & $-9,0$ & $-0,6$ \\
\hline CC2 & $\begin{array}{c}\text { AIE } 27359 \\
- \text { SIF } \\
20170090.015\end{array}$ & grande & tibia & $-18,2$ & $+9,5$ & 3,2 & $-10,0$ & $-6,3$ \\
\hline CC13 & AIE 27360 & grande & húmero & $-17,7$ & $+9,4$ & 3,3 & - & - \\
\hline CC14 & AIE 27361 & grande & fémur & $-17,5$ & $+8,8$ & 3,3 & - & - \\
\hline $\mathrm{CC} 15$ & AIE 27362 & grande & radio & $-17,4$ & $+8,5$ & 3,3 & - & - \\
\hline
\end{tabular}


Entonces, los valores de $\delta^{13} \mathrm{C}_{\mathrm{VPDB}}$ medidos sobre el colágeno de los camélidos de este sitio varían entre $-18,4 \%$ y $-17,1 \%$ y exhiben una media de $-17,8 \%$, mientras que los valores de $\delta^{15} \mathrm{~N}_{\mathrm{AIR}}$ se sitúan entre $+5,4 \%$ y $+9,5 \%$ y presentan un valor medio de $+7,9 \%$ o. Por otra parte, los valores de $\delta^{13} \mathrm{C}_{\mathrm{VPDB}}$ medidos sobre la fracción carbonato de la hidroxiapatita se sitúan entre $-10,6 \%$ o $-9,0 \%$ y presentan una media de $-9,8 \%$, mientras que los valores de $\delta^{18} \mathrm{O}_{\text {VPDB }}$ se encuentran entre $-6,3 \%$ y $-0,6 \%$ y exhiben una media de $-3,2 \%$. Dentro de las muestras de colágeno analizadas, ocho representan especímenes de tamaño grande, mientras que cuatro corresponden a especímenes de tamaño pequeño, presentando medias de $-17,8 \%$ y $+8,4 \%$ o en el primer caso y de $-17,9 \%$ y $+7,0 \%$ on el segundo para las composiciones isotópicas del carbono y del nitrógeno respectivamente (Figura 2). Por otra parte, dentro de las muestras de hidroxiapatita, cinco representan camélidos de tamaño grande al tiempo que cuatro corresponden a camélidos de tamaño pequeño, presentando medias de $-9,8 \%$ y $-3,1 \%$ o en el primer caso y de $-9,7 \%$ y $-3,3 \%$ en el segundo para los valores de $\delta^{13} \mathrm{C}_{\mathrm{VPDB}}$ y $\delta^{18} \mathrm{O}_{\mathrm{VPDB}}$ respectivamente (Figura 3).

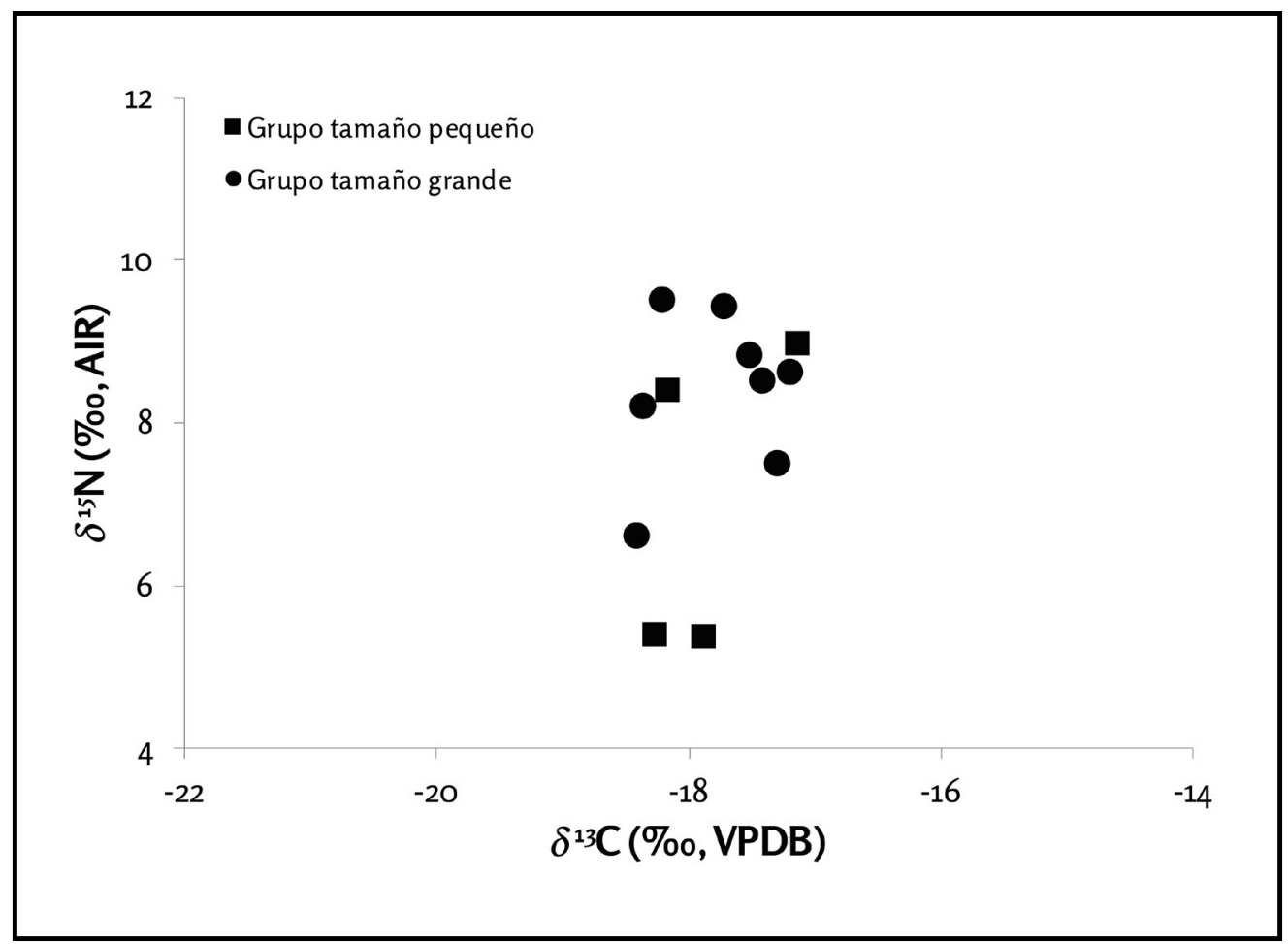

Figura 2. Valores de $\delta^{13} \mathrm{C}_{\mathrm{VPDB}}$ y $\delta^{15} \mathrm{~N}_{\mathrm{AIR}}$ medidos sobre colágeno extraído de restos óseos asignados a camélidos de los grupos de tamaño grande y pequeño recuperados en la capa 3 del sitio Cueva Chayal.

\section{Discusión}

A fin de interpretar los resultados obtenidos a partir del análisis isotópico de los huesos de camélidos recuperados en Cueva Chayal, decidimos evaluarlos en función de dos dimensiones de variabilidad: una vinculada con la información actual generada a partir del relevamiento de distintos materiales provenientes del área de estudio y otra ligada a la información osteométrica recabada a partir del análisis de las arqueofaunas del sitio. En el primer caso, podemos 


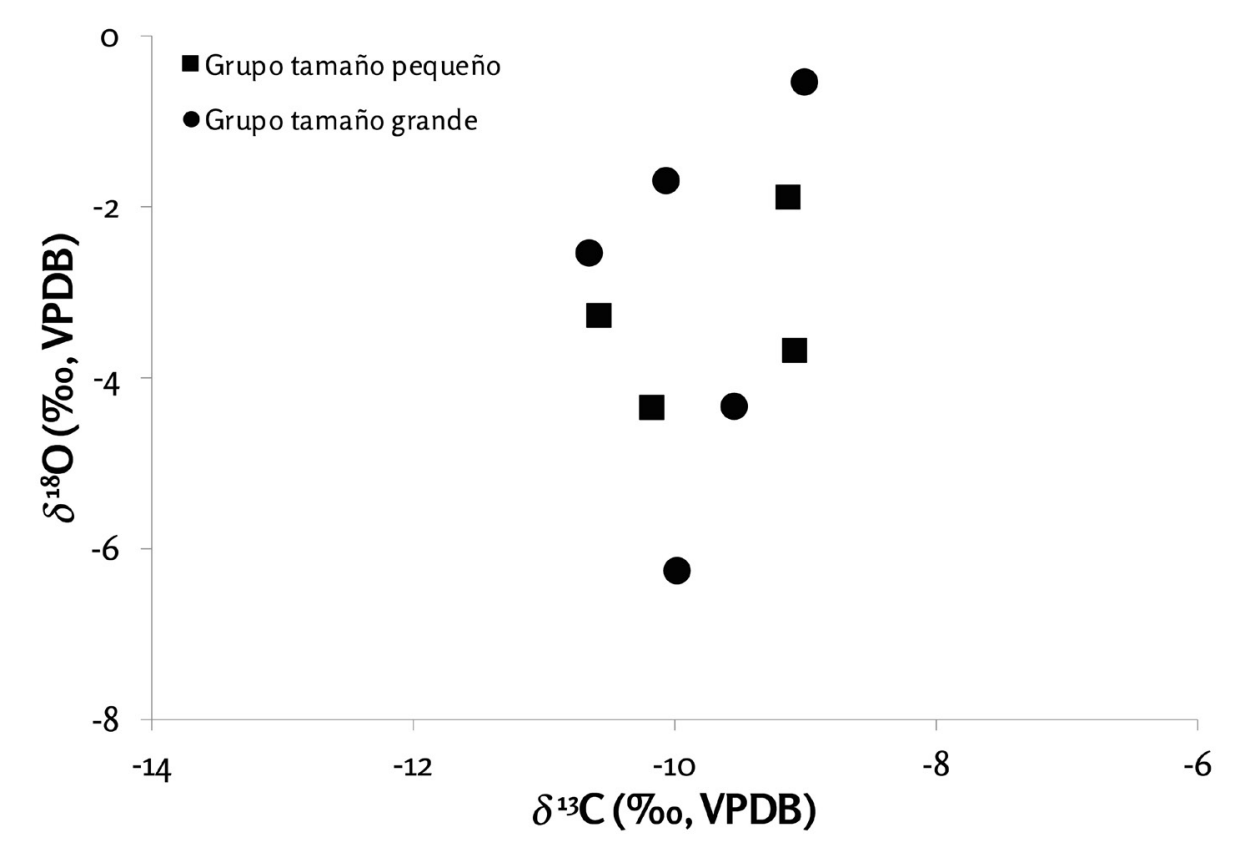

Figura 3. Valores de $\delta^{13} \mathrm{C}_{\mathrm{VPDB}}$ y $\delta^{18} \mathrm{O}_{\text {VPDB }}$ medidos sobre hidroxiapatita extraída de restos óseos asignados a camélidos de los grupos de tamaño grande y pequeño recuperados en la capa 3 del sitio Cueva Chayal.

relacionar nuestros resultados con aquellos obtenidos a partir de la medición de la composición isotópica de distintos materiales actuales del área de estudio, los cuales han permitido generar algunos modelos que vienen funcionando como líneas de base locales (Samec, Morales et al., 2017; Samec, Yacobaccio et al., 2017; Samec et al., 2018). En el segundo caso, podemos evaluar los datos en función de la asignación de cada espécimen en particular a uno u otro grupo de tamaño en representación de una especie silvestre y otra doméstica, en la medida en que consideramos como pertenecientes a la especie $V$. vicugna a los especímenes de tamaño pequeño y como parte de la especie L. glama a los especímenes de tamaño grande. Hasta el momento, hemos descartado la presencia de guanaco dentro del conjunto analizado, debido a que los ejemplares de tamaño grande que pudieron ser medidos se ubicaron en el extremo de mayor tamaño dentro del grupo grande, permitiendo situarlos dentro del espectro de tamańo "llama” (Yacobaccio et al., 1997-1998).

Dentro de la primera dimensión de análisis y a fin de estimar la proporción de vegetales $\mathrm{C}_{4}$ (y CAM) en la dieta de los camélidos muestreados aquí se decidió emplear el modelo de mezcla FRUITS (Fernandes, Millard, Brabec, Nadeau y Grootes, 2014). Para ello se tomaron las medias de las distintas vías fotosintéticas y sus respectivas desviaciones estándar en función de la información que fuera relevada a partir de la realización de censos de vegetación local, la cual se halla publicada en Samec (2014) y Samec, Yacobaccio et al. (2017). Al mismo tiempo, se aplicó un factor de corrección de $+1,5 \%$ a los valores actuales a fin de contrarrestar el llamado efecto Suess (Keeling, 1979) y se utilizó un factor de discriminación de 5 $\pm 1,3 \%$ para el car-

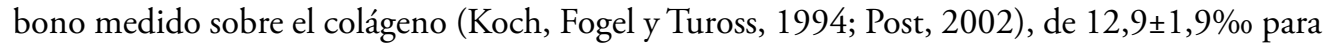
el carbono medido sobre la hidroxiapatita (Lee-Thorp, Sealy y Van der Merwe, 1989; Cerling 
y Harris, 1999) y de 3,4 $\pm 1 \%$ o para el nitrógeno medido sobre el colágeno (Post, 2002; Bocherens y Drucker, 2003). Entonces, el cálculo de la proporción de plantas $\mathrm{C}_{4}$ (y CAM) incluida en la dieta de los camélidos recuperados en la capa 3 de Cueva Chayal arroja un porcentaje comprendido entre 13 y $23 \%$ empleando todos los proxies con los que contamos $\left(\delta^{13} \mathrm{C}_{\mathrm{VPDB}} \mathrm{y}\right.$ $\delta^{15} \mathrm{~N}_{\mathrm{AIR}}$ medidos sobre colágeno y $\delta^{13} \mathrm{C}_{\mathrm{VPDB}}$ medidos sobre hidroxiapatita, $\mathrm{n}=9$ individuos), mientras que se encuentra entre 13 y $21 \%$ si solo consideramos los dos proxies medidos sobre colágeno $\left(\delta^{13} \mathrm{C}_{\mathrm{VPDB}} \mathrm{y} \delta^{15} \mathrm{~N}_{\mathrm{AIR}}, \mathrm{n}=12\right.$ individuos) (Tabla 4). La pequeña diferencia que surge al correr estos dos modelos puede deberse al aporte diferencial de una y otra vía fotosintética a la dieta proteica (medida en el colágeno) en comparación con la dieta total (medida en la hidroxiapatita) vinculada al enrutamiento diferencial de los diversos macronutrientes (Ambrose y Norr, 1993; Passey et al., 2005), aunque dicho enrutamiento diferencial no haya sido corroborado aún para camélidos sudamericanos.

Tabla 5. Proporción de vegetales $\mathrm{C}_{4}$ y CAM en la dieta de los camélidos analizados según el modelo de mezcla FRUITS (Fernandes et al., 2014).

\begin{tabular}{|c|c|c|c|c|c|c|}
\hline \multirow{2}{*}{$\begin{array}{l}\text { Proxies } \\
\text { Muestra }\end{array}$} & \multicolumn{3}{|c|}{$\begin{array}{c}\delta^{13} \mathrm{C}_{\mathrm{VPDB}} \mathbf{y} \\
\delta^{15} \mathrm{~N}_{\mathrm{AIR}} \text { sobre colágeno }\end{array}$} & \multicolumn{3}{|c|}{$\begin{array}{c}\delta^{13} \mathbf{C}_{\mathrm{VPDB}} \mathbf{y} \\
\delta^{15} \mathbf{N}_{\mathrm{AIR}} \text { sobre colágeno } \mathbf{y} \\
\delta^{13} \mathbf{C}_{\mathrm{VPDB}} \text { sobre apatita }\end{array}$} \\
\hline & $\begin{array}{c}\text { Proporción } \\
\text { media de } \\
\text { plantas } \mathrm{C}_{4} \\
\text { y CAM } \\
\text { en la dieta }\end{array}$ & $\begin{array}{l}\text { Desviación } \\
\text { estándar }\end{array}$ & $\begin{array}{l}\text { Intervalo } \\
\text { creíble del } \\
95 \%\end{array}$ & $\begin{array}{c}\text { Proporción } \\
\text { media de } \\
\text { plantas } \mathrm{C}_{4} \\
\text { y CAM } \\
\text { en la dieta }\end{array}$ & $\begin{array}{l}\text { Desviación } \\
\text { estándar }\end{array}$ & $\begin{array}{c}\text { Intervalo } \\
\text { creíble del } \\
95 \%\end{array}$ \\
\hline CC1 & 0,15 & 0,10 & $0,01-0,37$ & 0,18 & 0,10 & $0,02-0,38$ \\
\hline CC11 & 0,21 & 0,12 & $0,01-0,45$ & 0,20 & 0,11 & $0,02-0,42$ \\
\hline $\mathrm{CC} 3$ & 0,13 & 0,09 & $0,01-0,34$ & 0,13 & 0,09 & $0,01-0,38$ \\
\hline CC5 & 0,14 & 0,10 & $0,01-0,36$ & 0,14 & 0,09 & $0,01-0,33$ \\
\hline CC4 & 0,15 & 0,09 & $0,01-0,34$ & 0,13 & 0,07 & $0,01-0,28$ \\
\hline CC6 & 0,19 & 0,11 & $0,01-0,43$ & 0,20 & 0,09 & $0,02-0,37$ \\
\hline CC7 & 0,14 & 0,10 & $0,01-0,37$ & 0,15 & 0,09 & $0,01-0,33$ \\
\hline CC9 & 0,20 & 0,11 & $0,02-0,44$ & 0,23 & 0,11 & $0,02-0,47$ \\
\hline $\mathrm{CC} 2$ & 0,16 & 0,11 & $0,01-0,39$ & 0,19 & 0,10 & $0,02-0,40$ \\
\hline $\mathrm{CC} 13$ & 0,18 & 0,11 & $0,01-0,41$ & & & \\
\hline CC14 & 0,20 & 0,11 & $0,01-0,43$ & & & \\
\hline CC15 & 0,20 & 0,12 & $0,01-0,45$ & & & \\
\hline
\end{tabular}

En relación a la proporción de vegetales $\mathrm{C}_{4}$ en la dieta de los camélidos muestreados y su correlación con la variable altitudinal, podemos destacar que los valores de $\delta^{13} \mathrm{C}_{\mathrm{VPDB}}$ medidos sobre colágeno extraído de los materiales óseos recuperados en el sitio se ajustan al modelo altitudinal elaborado en función de los datos modernos para llamas y vicuñas (Samec, 2014; Samec et al., 2018), aunque se manifiestan un tanto por debajo de lo esperado, indicando un mayor aporte 
de plantas $\mathrm{C}_{3}$ en la dieta que el esperado según la cota altitudinal en la que se localiza el sitio (Figura $4 \mathrm{~A}$ y C). Este ligero desajuste con relación al modelo actual puede deberse a las características propias de la quebrada de Chayal ligadas a sus condiciones de humedad proclives al desarrollo de vegetación $\mathrm{C}_{3}$ en relación con las comunidades circundantes y/o a la existencia de variaciones en cuanto al uso de los espacios de pastoreo y caza en el pasado, los cuales podrían haber incluido parches localizados a mayor altitud y con una mayor abundancia relativa de especies $\mathrm{C}_{3}$, a lo que también podría haberse sumado una modificación de los arreglos vegetacionales en los distintos parches dictada por cambios ambientales. Por otra parte, al observar los valores de $\delta^{15} \mathrm{~N}_{\text {AIR }}$ medidos sobre el colágeno extraído de las arqueofaunas de Cueva Chayal, vemos que estos se ajustan un poco mejor a lo esperado en función de la correlación existente entre la altitud y los valores de $\delta^{15} \mathrm{~N}_{\mathrm{AIR}}$ para camélidos modernos muestreados en el área de estudio (Samec et al., 2018) (Figura 4B y D). Este último patrón parece abogar a favor de la inexistencia de transformaciones remarcables en los arreglos de las comunidades vegetacionales utilizadas en función de las variaciones ambientales y/o la inexistencia de grandes movimientos en el traslado de los rebańos y/o en el uso de cotos de caza.
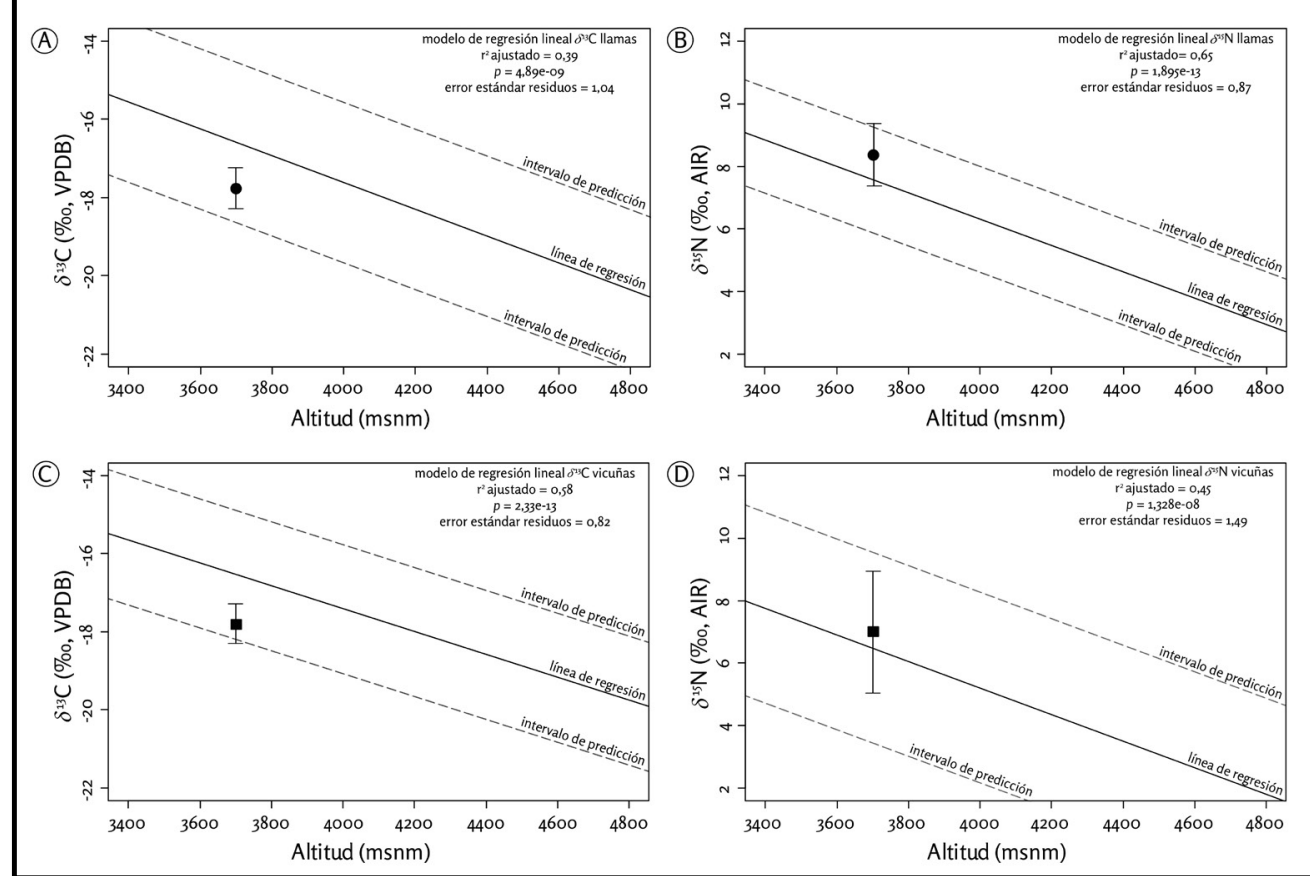

Figura 4. Representación de las medias (y desviaciones estándar) de los valores isotópicos medidos sobre colágeno óseo de los materiales recuperados en Cueva Chayal en relación con los modelos de regresión lineal según la altitud, creados a partir del análisis de materiales modernos.

A) Media (y desviación estándar) del grupo grande según el modelo de regresión lineal para valores de $\delta^{13} \mathrm{C}_{\mathrm{VPDB}}$ de llamas modernas, B) Media (y desviación estándar) del grupo grande según el modelo de regresión lineal para valores de $\delta^{15} \mathrm{~N}_{\text {AIR }}$ de llamas modernas, C) Media (y desviación estándar) del grupo pequeño según el modelo de regresión lineal para valores de $\delta^{13} \mathrm{C}_{\mathrm{VPDB}}$ de vicuñas modernas, y D) Media (y desviación estándar) del grupo pequeño según el modelo de regresión lineal para valores de $\delta^{15} \mathrm{~N}_{\text {AIR }}$ de vicuñas modernas (ver Samec et al., 2018 para mayores detalles). 
En el caso de los valores de $\delta^{18} \mathrm{O}_{\mathrm{VPDB}}$, se tomaron las ecuaciones utilizadas por Goepfert et al. (2013) basadas a su vez en Amiot et al. (2004) y Lécuyer et al. (2010) a fin de estimar la composición isotópica del agua ingerida y establecer un correlato con los valores de precipitaciones estimados a partir del OIPC (Online Isotopes in Precipitation Calculator) (Bowen, 2017) en función de los antecedentes de la base de datos GNIP (Global Network for Isotopes in Precipitation) (IAEA/WMO, 2015) y los valores medidos en aguas superficiales en cercanías al sitio en cuestión (Samec, Morales et al., 2017). A partir de ello se observa que, si bien algunos valores de $\delta^{18} \mathrm{O}_{\text {vSMOW }}$ del agua ingerida por los camélidos del sitio se ajustan a lo esperado en función de lo estimado por el OIPC, otros caen por fuera de lo esperado en función de esta herramienta y muestran valores más elevados (Figura 5). Considerando que el relevamiento actual de los recursos acuíferos disponibles ha demostrado que existen acumulaciones naturales de agua de lluvia sujetas a altas tasas de evaporación que exhiben valores de $\delta^{18} \mathrm{O}_{\text {vsmow }}$ positivos (Samec, Morales et al., 2017), proponemos que el patrón identificado responde a la ingesta de aguas evaporadas por parte de los camélidos muestreados. Asimismo, vale destacar que los restos óseos muestreados aquí corresponden a una acumulación de al menos 270 años (en función de las diferencias entre los fechados obtenidos en la capa 3 de la cueva), representando distintos eventos de desposte y consumo de camélidos, tanto silvestres como domesticados. Por tanto, es factible que la variabilidad exhibida por los valores estimados de $\delta^{18} \mathrm{O}_{\text {vsMow }}$ para el agua ingerida responda a la variabilidad de los recursos acuíferos utilizados por los rebaños de llamas y los grupos de vicuńas durante ese lapso, expresando no solo variaciones espaciales en función de la localización y naturaleza de los recursos acuíferos utilizados, sino también variaciones climáticas en escalas estacionales, anuales y decadales en función de la variabilidad

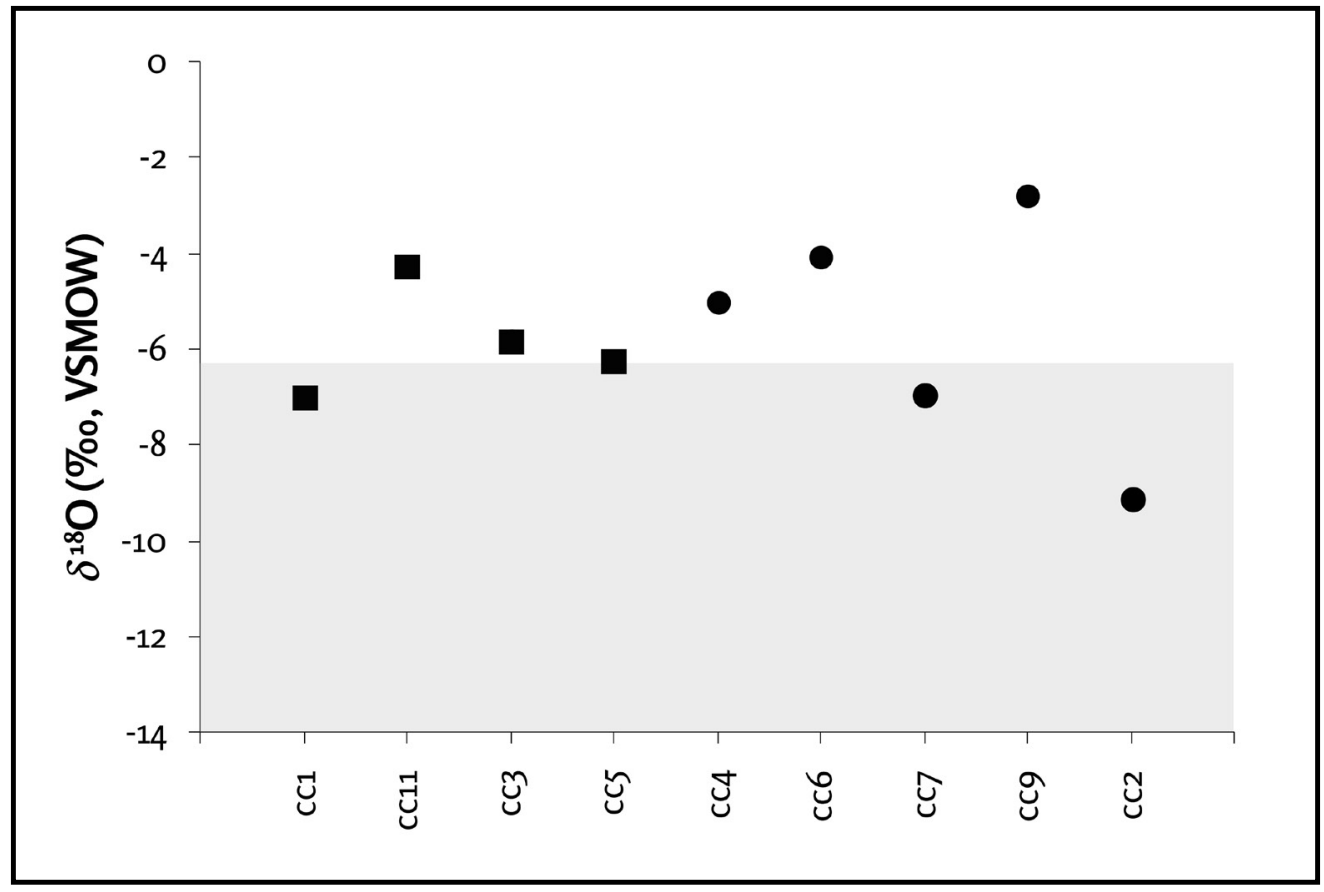

Figura 5. Valores estimados de $\delta^{18} \mathrm{O}_{\mathrm{VSMOW}}$ del agua ingerida por parte de camélidos del grupo pequeño (cuadrados) y del grupo grande (círculos).

El área del gráfico sombreada corresponde a los valores de $\delta^{18} \mathrm{O}_{\text {VSMOw }}$ estimados para las precipitaciones de enero a diciembre para la localización de Cueva Chayal según el OIPC (Online Isotopes in Precipitation Calculator) (Bowen, 2017). 
de las precipitaciones en el área de estudio (Morales, Carilla, Grau y Villalba, 2015). Al mismo tiempo, debemos recordar que la secuencia paleoclimática de alcance regional establecida según los datos recabados en la cercana Laguna Pululos indica la existencia de un momento árido entre 1310 y 1540 DC, con un pico de aridez alrededor de los 1400 DC (Lupo et al., 2007), un aspecto que pudo haber alterado la composición isotópica de las aguas meteóricas y/o de las acumulaciones de agua disponibles en superficie, pudiendo presentar valores de $\delta^{18} \mathrm{O}_{\text {vSMOw }}$ más elevados a raíz del incremento en las temperaturas medias en la región.

Ahora bien, ya dentro de la segunda dimensión de variabilidad mencionada más arriba, nuestros resultados no muestran diferencias en la proporción de vegetales $\mathrm{C}_{3} \mathrm{y} \mathrm{C}_{4}$ incluidos en la dieta de camélidos domésticos y silvestres, ya sea que observemos los valores de $\delta^{13} \mathrm{C}_{\mathrm{VPDB}}$ medidos sobre colágeno o hidroxiapatita (Figuras 6 y 7, Tabla 4). Por otro lado, los resultados muestran diferencias en los valores de $\delta^{15} \mathrm{~N}_{\text {AIR }}$ medidos sobre el colágeno de las distintas especies de camélidos consideradas, las cuales no tienen un correlato en los valores de $\delta^{13} \mathrm{C}_{\mathrm{VPDB}}$ de las mismas. Esta información se encuentra en consonancia con los patrones identificados al analizar las composiciones isotópicas de camélidos domésticos y silvestres en contextos actuales, tal como fuera indicado por Samec et al. (2018). En este sentido, resulta notable que las muestras del sitio correspondientes a camélidos de tamańo grande presenten valores de $\delta^{15} \mathrm{~N}_{\mathrm{AIR}}$ más elevados que los camélidos de tamaño pequeño, imitando el patrón que encontramos en la actualidad al comparar llamas con vicuñas, aunque presentando una notable variabilidad. Por otra parte, al observar los valores de $\delta^{18} \mathrm{O}_{\text {VPDB }}$ encontramos que aquellos datos correspondientes a especímenes de tamaño grande y asignados a L. glama presentan una variabilidad mayor que aquellos asignados a V. vicugna. Este patrón podría responder al carácter de "acumulación" del conjunto mencionado más arriba o bien ser el producto del uso alternado de diferentes áreas de pastoreo y, por consiguiente, diferentes fuentes de agua en oposición al comportamiento más "territorial" de la especie silvestre y el consiguiente establecimiento de áreas de caza ancladas a esos territorios y sus recursos acuíferos.
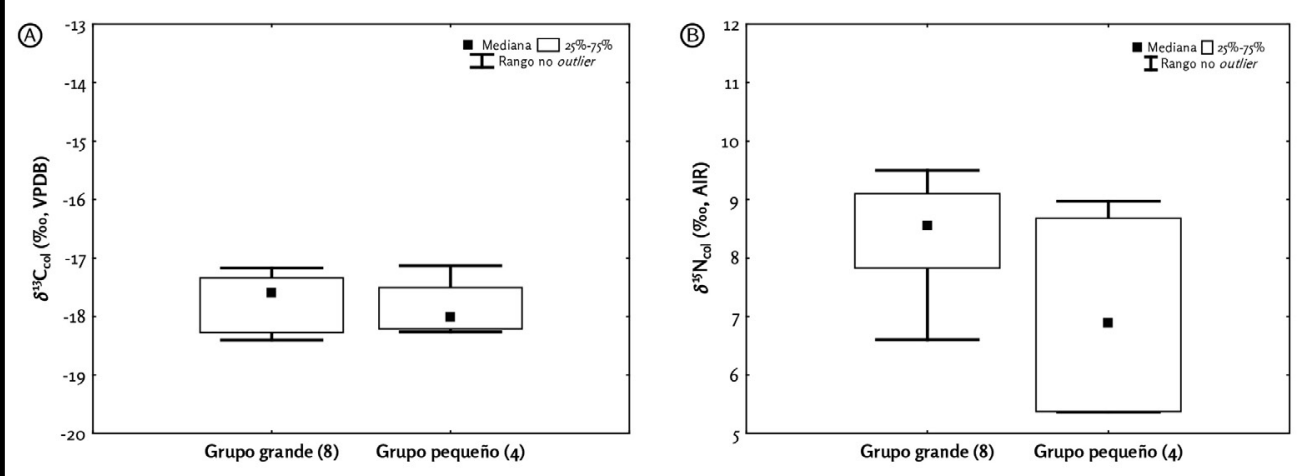

Figura 6. Valores de $\delta^{13} \mathrm{C}_{\mathrm{VPDB}}$ y $\delta^{15} \mathrm{~N}_{\mathrm{AIR}}$ medidos sobre colágeno óseo según grupo de tamaño. 


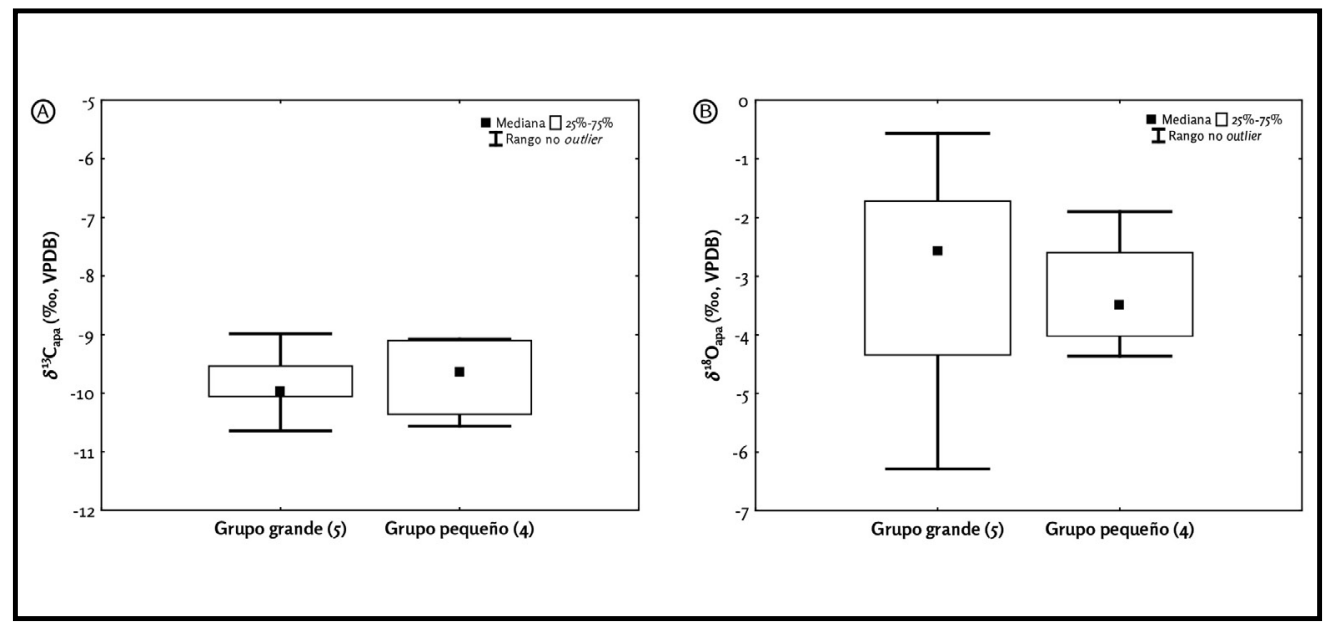

Figura 7. Valores de $\delta^{13} \mathrm{C}_{\mathrm{VPDB}}$ у $\delta^{18} \mathrm{O}_{\mathrm{VPDB}}$ medidos sobre hidroxiapatita ósea según grupo de tamaño.

Para finalizar, entonces, nos interesa recapitular sobre las nuevas evidencias que arrojan los análisis isotópicos realizados sobre las arqueofaunas del sitio Cueva Chayal. En primera instancia resulta relevante verificar el ajuste de los valores isotópicos de $\delta^{13} \mathrm{C}_{\mathrm{VPDB}}$ (medidos tanto sobre colágeno como hidroxiapatita) de los materiales arqueofaunísticos con respecto a los valores isotópicos de los materiales actuales y las líneas de base que se han establecido para la región de estudio (Samec, Yacobaccio et al., 2017; Samec et al., 2018). Este aspecto parece apuntar a la existencia de un patrón de uso del espacio y áreas de pastoreo de los rebaños semejante al que existe en la actualidad, con ligeras variaciones -evidenciadas, por ejemplo, en un porcentaje de vegetación $\mathrm{C}_{3}$ en la dieta ligeramente más elevado que el esperado- aunque dentro de los parámetros establecidos. En el caso de los valores de $\delta^{15} \mathrm{~N}_{\text {AIR }}$, estos también se ajustan a lo esperado según el modelo altitudinal y no parecen indicar la existencia de grandes cambios climáticos para el momento considerado, en la medida en que el balance de nitrógeno disponible en el ambiente no parece haberse visto afectado (algo de lo que sí habría evidencias durante el Holoceno Medio (ver Samec et al., 2014 para mayores detalles). Asimismo, los valores de $\delta^{18} \mathrm{O}_{\text {VPDB }}$ medidos sobre hidroxiapatita presentan una gran variabilidad, que no en todos los casos se ajusta a lo esperado en función de las precipitaciones medidas en el área de estudio, aunque sí lo hace si se consideran otros recursos como espejos de agua sujetos a evaporación excesiva (Samec, Morales et al., 2017). Sin embargo, los datos generados hasta el momento en materia de valores de $\delta^{18} \mathrm{O}_{\mathrm{VPDB}}$ y su potencial como indicador de movimientos altitudinales y/o variaciones en los regímenes de precipitación aún no resultan del todo claros y merecen un estudio específico sobre el tema. Finalmente, en lo que se refiere a la variación de los valores isotópicos en función de las categorías de tamaño -que fueran empleadas aquí como categorías taxonómicas-, no encontramos una tendencia clara en las composiciones isotópicas analizadas que nos permitan señalar la existencia de áreas diferenciables utilizadas en las actividades de caza y pastoreo. La existencia de áreas "comunes" para ambas actividades resulta factible y puede fundamentarse en el hecho de que aún hoy en día se puede observar a vicuñas y llamas pastando en simpatría en los mismos parches vegetacionales (Arzamendia y Vilá, 2014). Por esto, puede postularse que no necesariamente existió una estricta separación de las áreas de pastoreo y caza, al tiempo que estrategias de caza silenciosas - p.e., aquella practicada con arco y flecha a corta distancia- llevadas a cabo de manera esporádica y de forma individual no producirían grandes alteraciones (huida) en el comportamiento de los camélidos silvestres 
(Yacobaccio y Vilá, 2016). Mención aparte merecen los valores de $\delta^{15} \mathrm{~N}_{\mathrm{AIR}}$ de ambos grupos de tamaño, los cuales muestran la existencia de una diferencia similar a la que se manifiesta al comparar llamas con vicuńas en la actualidad (Samec et al., 2018), aspecto que parece corroborar nuestra clasificación taxonómica de las arqueofaunas del sitio, aunque aún resta identificar qué factores determinan la existencia de este patrón tanto en los resultados actuales como en los arqueológicos. De cualquier manera, la presencia de especímenes óseos asignados a llamas y vicuñas con valores isotópicos semejantes y consistentes con las líneas de base propuestas para el área de estudio nos informa sobre la existencia de una estrategia de subsistencia que abarcaba tanto el pastoreo de camélidos domésticos como la caza de camélidos silvestres y otras especies -Lagidium sp.- disponibles en las cercanías de la cueva. En este sentido, la adopción del pastoreo de camélidos domésticos como actividad predominante no habría operado en desmedro de las prácticas de caza, sino que estas se habrían integrado dentro una estrategia de subsistencia basada en diversos recursos, permitiendo garantizar la viabilidad reproductiva de los rebaños al reducir la presión sobre los mismos, incluso en contextos de elevado riesgo ambiental como el que representa la puna de Atacama durante el Holoceno Tardío (Yacobaccio et al., 1997-1998).

\section{Palabras finales}

Los datos isotópicos generados a partir del análisis de las muestras óseas de camélidos domésticos y silvestres provenientes del sitio Cueva Chayal se ajustan en mayor o menor medida a lo esperado en función de los datos actuales con los que contamos (Yacobaccio et al., 2009; Samec, 2014; Samec, Morales et al., 2017; Samec, Yacobaccio et al., 2017; Samec et al., 2018). Este aspecto, sumado a la información zooarqueológica que fuera recabada sobre los materiales del sitio, permitiría considerar la cueva como parte de un circuito pastoril que involucraba locaciones diferentes en función de la disponibilidad estacional de pasturas, tal como sucede en la época actual (Yacobaccio, Madero y Malmierca, 1998; Yacobaccio, 2007). Vale mencionar que la conformación del paisaje local compuesto por planicies disectadas por profundos cañadones modifica las características de los arreglos vegetacionales en distancias relativamente cortas. Por lo tanto, la alternancia de pasturas en esta área pudo haber involucrado el traslado de los rebańos en pequeńas distancias relativas de alrededor de 5-10 km lineales, involucrando la vegetación propia de la quebrada y aquella de la estepa mixta situada en las elevaciones circundantes a esta.

Sin embargo, existe un problema de equifinalidad en la interpretación de nuestros resultados dado que resulta imposible establecer en qué medida las (mayores o menores) diferencias existentes entre lo esperado según la línea de base actual y los valores isotópicos medidos sobre las arqueofaunas responden a los cambios ambientales propios de este tramo del Holoceno tardío y/o de la existencia de estrategias particulares en el uso del espacio y los recursos circundantes implementadas por los pastores en el pasado. Por lo pronto, consideramos que la información reunida en este trabajo, sumada a la identificación de un patrón de asentamiento disperso y a las características de los modos de explotación faunística, indican la existencia de una estrategia de pastoreo de camélidos domésticos complementada a partir de la caza de animales silvestres (tanto camélidos como roedores) en el área de estudio durante el período considerado (ca. 1300-1500 DC). Esta estrategia se extendió por lo menos durante los últimos dos mil años en el área de Susques y representa el corolario del proceso de domesticación de los camélidos sudamericanos que tuviera lugar en las tierras altas del área centro-sur andina (Yacobaccio et al., 1997-1998). Al mismo tiempo, vale destacar que los datos obtenidos a partir del análisis 
de isótopos estables de restos humanos hallados en sitios cercanos a Cueva Chayal y con una cronología similar (p.e., Hornillos 3 o Chulpa Chayal) se muestran consistentes con la información recabada a partir del análisis de la fauna de la cueva, en la medida que la dieta de estos individuos involucraba el consumo de camélidos con valores similares a los registrados, además de otros recursos (Yacobaccio et al., 1997-1998). Finalmente, nuevas investigaciones deberán explorar la influencia del cambio climático en el largo y el mediano plazo, así como las oscilaciones estacionales, dentro de la variación observada en los valores isotópicos medidos sobre las arqueofaunas de Cueva Chayal y otros sitios contemporáneos dentro del área de estudio.

\section{Agradecimientos}

Queremos expresar nuestro agradecimiento a la comunidad de Susques, que nos recibió con los brazos abiertos durante tantos años de trabajo en el campo. Agradecemos también al personal del Instituto de Geocronología y Geología Isotópica de Buenos Aires y del Laboratorio de Isótopos Estables en Ciencias Ambientales de San Rafael por su colaboración en el pretratamiento de las muestras analizadas. Destacamos la colaboración de Augusto Tessone, Malena Pirola y Francisca Santana Sagredo, quienes resolvieron algunas dudas durante la preparación del manuscrito. Y reconocemos el aporte de dos evaluadores anónimos cuyos comentarios permitieron mejorar el escrito. Este trabajo fue financiado por la Agencia Nacional de Investigación y Desarrollo de Chile (Proyecto postdoctoral FONDECYT folio 3190566), el Consejo Nacional de Investigaciones Científicas y Técnicas de Argentina (Proyecto PIP 0853) y la Universidad de Buenos Aires (Proyecto UBACyT F-383).

\section{Referencias citadas}

Ambrose, S. H. y Norr, L. (1993). Experimental evidence for the relationship of the carbon isotope ratios of whole diet and dietary protein to those of bone collagen and carbonate. En Lambert, J. B. y Norr, L. (Eds.). Prehistoric Human Bone - Archaeology at the Molecular Level (pp. 1-38). Berlín: Springer-Verlag.

Amiot, R., Lecuyer, C., Buffetaut, E., Fluteau, F., Legendre, S. y Martineau, F. (2004). Latitudinal temperature gradient during the Cretaceous Upper Campanian-Middle Maastrichtian: $\delta^{18} \mathrm{O}$ record of continental vertebrates. Earth and Planetary Science Letters, 226, 255-272.

Arzamendia, Y., y Vilá, B. (2014). Vicugna habitat use and interactions with domestic ungulates in Jujuy, Northwest Argentina. Mammalia, 79(3), 267-278.

Behrensmeyer, A. (1978). Taphonomic and ecologic information from bone weathering. Paleobiology, 4, 150162.

Bianchi, A. R., Yañez, C. E. y Acuña, L. R. (2005). Bases de datos mensuales de las precipitaciones del Noroeste Argentino. Informe del Proyecto Riesgo Agropecuario. INTA-SAGPYA.

Bocherens, H. y Drucker, D. (2003). Trophic level isotopic enrichment of carbon and nitrogen in bone collagen: case studies from recent and ancient terrestrial ecosystems. International Journal of Osteoarchaeology, $13,46-53$.

Bowen, G. J. (2017). The Online Isotopes in Precipitation Calculator, version 3.1. http://www.waterisotopes.org.

Butt, B. (2010). Seasonal space-time dynamics of cattle behavior and mobility among Maasai pastoralists in semi-arid Kenya. Journal of Arid Environments, 74, 403-413. 
Cabrera, A. L. (1976). Regiones fitogeográficas argentinas. Enciclopedia Argentina de Agricultura y jardinería. Tomo II ( $2^{a}$ ed.). Buenos Aires: Editorial Acme.

Capriles Flores, J. M. y Tripcevich, N. (Eds.). (2016). The archaeology of Andean pastoralism. Albuquerque, NM: University of New Mexico Press.

Cartajena, I. (2013). Faunal assemblages from the middle Holocene environmental and cultural variability in the western slope of the puna de Atacama. Quaternary International, 307, 31-37.

Cartajena, I., Núnez, L. y Grosjean, M. (2007). Camelid domestication in the western slope of the Puna de Atacama, Northern Chile. Anthropozoologica, 42,155-173.

Cerling, T. E. y Harris J. M. (1999). Carbon isotope fractionation between diet and bioapatite in ungulate mammals and implications for ecological and paleoecological studies. Oecologia, 120, 347-363.

Crowley, B. E. y Wheatley, P. V. (2014). To bleach or not to bleach? Comparing treatment methods for isolating biogenic carbonate. Chemical Geology, 381, 234-242.

Dansgaard, W. (1964). Stable isotopes in precipitation. Tellus, 16, 436-468.

Dantas, M. (2012). Identificación interespecífica de camélidos en el valle de Ambato (Catamarca, Argentina). Una aproximación a la problemática desde distintas líneas de análisis. Revista del Museo de Antropología, 5(1), 259-268.

Dantas, M., Figueroa, G. G. y Laguens, A. (2014). Llamas in the Cornfield: Prehispanic Agro-Pastoral System in the Southern Andes. International Journal of Osteoarchaeology, 24, 149-165.

Dantas, M., y Knudson, K. J. (2016). Isótopos de estroncio: cría, circulación y apropiación de camélidos en Aguada de Ambato (Catamarca, Argentina). Intersecciones en Antropología, 17, 239-250.

De Niro, M. (1985). Postmortem preservation and alteration of in vivo bone collagen isotope ratios in relation to palaeodietary reconstruction. Nature, 317, 806-809.

Dransart, P. Z. (1991). Llamas, herders and the exploitation of raw materials in the Atacama Desert. World Archaeology, 22(3), 304-319.

Dransart, P. Z. (2011). Social principles of Andean camelid pastoralism and archaeological interpretations. En Albarella, U. y Trentacoste, A. (Eds.) Ethnozooarchaeology: The Present and Past of Human-Animal Relationships (pp. 123-130). Oxford: Oxbow Books.

Dufour, E., Goepfert, N., Léon, B. G., Chauchat, C., Jordán, R. F. y Sánchez, S. V. (2014). Pastoralism in Northern Peru during pre-Hispanic times: Insights from the Mochica Period (100-800 AD) based on stable isotopic analysis of domestic camelids. PloS one, 9(1), e87559.

Elkin. D. C. (1995). Volume density of South American camelids skeletal parts. International Journal of Osteoarchaeology, 5, 29-37.

Elkin, D. C. y Rosenfeld, S. (2001). La explotación de camélidos en Pintoscayoc 1 (Jujuy). En Mengoni Goñalons, G. L., Olivera, D. E. y Yacobaccio, H. D. (Eds.). El uso de los camélidos a través del tiempo (pp. 29-64). Buenos Aires: Ediciones del Tridente.

Fernandes, R., Millard, A. R., Brabec, M., Nadeau, M. J., Grootes, P. (2014). Food Reconstruction Using Isotopic Transferred Signals (FRUITS): A Bayesian Model for Diet Reconstruction. Plos One, 9(2), e87436. 
Fernández, J. y Panarello, H. O. (1989-1990). Isótopos en Arqueología. 1. Valores isotópicos del oxígeno en aguas meteóricas y su pasaje a la sangre humana y a la de camélidos de la Puna Jujeña a $4.000 \mathrm{~m}$ de altitud. Runa, XIX, 47-58.

Fernández, J. y Panarello, H. O. (1999-2001). Isótopos del carbono en la dieta de herbívoros y carnívoros de los Andes Jujeños. Xama, 12-14, 71-85.

Finucane, B. C., Maita Agurto, P. e Isbell, W. H. (2006). Human and animal diet at Conchopata, Perú: stable isotope evidence for maize agriculture and animal management practices during the Middle Horizon. Journal of Archaeological Science, 33, 1766-1776.

Gallardo, F. y Yacobaccio, H. (2005). Wild or domesticated? Camelids in early formative rock art of the Atacama Desert (Northern Chile). Latin American Antiquity, 16(2), 115-130.

Goepfert, N., Dufour, E., Gutiérrez, B. y Chauchat, C. (2013). Origen geográfico de camélidos en el periodo mochica (100-800 AD) y análisis isotópico secuencial del esmalte dentario: enfoque metodológico y aportes preliminares. Bulletin de l'Institut Français d'Etudes Andines, 42, 25-48.

Grant, J., Mondini, M. y Panarello, H. O. (2018). Carbon and nitrogen isotopic ecology of Holocene camelids in the Southern Puna (Antofagasta de la Sierra, Catamarca, Argentina): Archaeological and environmental implications. Journal of Archaeological Science: Reports, 18, 637-647.

Grant, J. y Olivera, D. (2016). Isótopos estables, movilidad y camélidos en sociedades agropastoriles tempranas de la Puna Meridional Argentina. Arqueología, 22. Dossier, 13-35.

Hogg, A., Heaton, T., Hua, Q., Palmer, J., Turney, C., Southon, J., Bayliss, A., Blackwell, P., Boswijk, G., Bronk Ramsey, C., Petchey, F., Reimer, P., Reimer, R., y Wacker, L. (2020). SHCal20 Southern Hemisphere calibration, 0-55,000 years cal BP. Radiocarbon, 1-20.

IAEA/WMO (2015). Global Network of Isotopes in Precipitation. The GNIP Database. Accessible at: https:// nucleus.iaea.org/wiser.

Izeta, A. D., Laguens, A. G., Marconetto, M. B. y Scattolin, M. C. (2009). Camelid handling in the meridional Andes during the first millennium AD: a preliminary approach using stable isotopes. International Journal of Osteoarchaeology, 19, 204-214.

Keeling, C. D. (1979). Recent trends in the ${ }^{13} \mathrm{C} /{ }^{12} \mathrm{C}$ ratio of atmospheric carbon dioxide. Nature, 277, 121-122.

Kent, J. D. (1982). The Domestication and Exploitation of the South American Camelids: Methods of Analysis and Their Application to Circum-Lacustrine Archaeological Sites in Bolivia and Peru. Tesis doctoral. Washington University-St. Louis. University Microfilms.

Khazanov, A. M. (1994). Nomads and the Outside World. Madison, WI: University of Wisconsin Press.

Knudson, K. J. (2009). Oxygen isotope analysis in a land of environmental extremes: the complexities of isotopic work in the Andes. International Journal of Osteoarchaeology, 19(2), 171-191.

Knudson, K. J., Gardella, K. R. y Yaeger, J. (2012). Provisioning Inka feasts at Tiwanaku, Bolivia: the geographic origins of camelids in the Pumapunku complex. Journal of Archaeological Science, 39(2), 479-491.

Koch, P. L., Fogel, M. L. y Tuross, N. (1994). Tracing the diets of fossil animals using stable isotopes. En: Stable isotopes in ecology and environmental Science (pp. 63-92). Oxford: Blackwell Scientific Publications. 
Laity, J. (2008). Deserts and Desert Environments. Chichester: Willey-Blackwell.

Lécuyer, C., Balter, V., Martineau, F., Fourel, F., Bernard, A., Amiot, R., Gardien, V., Otero, O., Legendre, S., Panczer, G., Simon, L. y Martini, R. (2010). Oxygen isotope fractionation between apatite-bound carbonate and water determined from controlled experiments with synthetic apatites precipitated at $10^{\circ} \mathrm{C}$ to $37^{\circ} \mathrm{C}$. Geochimica et Cosmochimica Acta, 74, 2072-2081.

Lee-Thorp, J., Sealy, J. y van der Merwe, N. (1989). Stable carbon isotope ratio differences between bone collagen and bone apatite, and their relationship to diet. Journal of Archaeological Science, 32, 1459-1470.

López, G. (2003). Pastoreo y caza en el Temprano de la Puna de Salta: Datos osteométricos del sitio Matancillas 2. Intersecciones en Antropología, 4, 17-27.

López, P., Cartajena, I. y Núñez, L. (2013). Análisis de isótopos estables en colágeno de huesos de camélidos de Quebrada Tulán, Puna de Atacama, Periodo Formativo Temprano (ca.3100-2400 AP). Chungara. Revista de Antropología Chilena, 45, 237-247.

López, P., Cartajena, I., Loyola, R., Núneez, L. y Carrasco, C. (2017). The use of hunting and livestock space inferred by stable isotopes analyses on camelids during the early Formative (Tilocalar Phase) in Tulan Transect (Puna de Atacama, Chile). International Journal of Osteoarchaeology, 27(6),1059-1069.

Lupo, L., Morales, M. R., Yacobaccio, H. D., Maldonado, A. y Grosjean, M. (2007). Cambios ambientales en la Puna Jujeńa durante los últimos 1200 ańos: explorando su impacto en la economía pastoril. Pacarina, III, 151-156.

Makarewicz, C. A. (2013). A pastoralist manifesto: Breaking stereotypes and re-conceptualizing pastoralism in the Near Eastern Neolithic. Levant, 45(2), 159-174.

Mengoni Gońalons, G. L. (2007). Camelid management during Inca times in N. W. Argentina: models and archaeozoological indicators. Anthropozoologica, 42(2), 129-141.

Mengoni Goñalons, G. L. (2014). Isótopos estables en camélidos del Período Tardío del Noroeste de Argentina (NOA): Estrategias de uso y manejo de rebaños. Revista Chilena de Antropología, 30, 68-75.

Mengoni Goñalons, G. L. y Yacobaccio, H. D. (2006). The domestication of South American camelids. A view from the South-Central Andes. En Zeder, M. A., Bradley, D. G., Emshwiller, E. y Smith, B. D. (Eds.). Documenting domestication. New Genetic and Archaeological Paradigms (pp. 228-244). University of California Press.

Mondini, M., Grant, J., Panarello, H. O., Samec, C., López M., P., Núñez, L. y Cartajena, I. (2019). Composición isotópica del carbono y el nitrógeno en camélidos arqueológicos de la Puna de Atacama: una comparación entre ambas vertientes de los Andes. Cuadernos del Instituto Nacional de Antropología y Pensamiento Latinoamericano, Series Especiales 7(2),172-181.

Mondini, M., Panarello, H. y Zangrando, A. (2010). First isotopic evidence in southern Puna camelids throughout the Holocene. Trabajo presentado en la $11^{\text {th }}$ Conference of the International Council for Archaeozoology. París, Francia.

Mondini, M. y Panarello, H. (2014). Isotopic evidence in Holocene camelids from the southern Puna. Trabajo presentado en la $12^{\text {th }}$ Conference of the International Council for Archaeozoology. San Rafael, Argentina.

Moore, K. (2016). Early Domesticated Camelids in the Andes. En Capriles, J. y Tripcevich, N. (Eds.). The Archaeology of Andean Pastoralism (pp. 17-38). Albuquerque, NM: University of New Mexico Press. 
Morales, M. R., Barberena, R., Belardi, J., Borrero, L., Cortegoso, V., Durán, V., Guerci, A., Goñi, R., Gil, A., Neme, G., Yacobaccio, H. y Zárate, M. (2009). Reviewing Human-Environment Interactions in Arid Regions of Southern South America During the Past 3000 Years. Palaeogeography, Palaeoclimatology, Palaeoecology, 281(3-4), 283-295.

Morales, M. S., Carilla, J., Grau, H. R. y Villalba, R. (2015). Multi-century lake area changes in the Andean high-elevation ecosystems of the Southern Altiplano. Climate of the Past, 11, 1139-1152.

Morales, M. S., Christie, D. A., Villalba, R., Argollo, J., Pacajes, J., Silva, J. S., Álvarez, C. A., Llancabure, J. C. y Soliz Gamboa, C. C. (2012). Precipitation changes in the South American Altiplano since 1300 AD reconstructed by tree-rings. Climate of the Past, 8, 653-666,

Olivera, D. E. (1997). La importancia del Recurso Camelidae en la Puna de Atacama entre los 10.000 y 500 años A.P. Estudios Atacameños, 14, 29-41.

Olivera, D. E. y Elkin, D. C. (1994). De cazadores y pastores: El proceso de domesticación de camélidos en la Puna Meridional Argentina. En Elkin, D. C., Madero, C., Mengoni Goñalons, G. L., Olivera, D. E., Reigadas, M. C. y Yacobaccio, H. D. (Eds.). Zooarqueología de camélidos 1 (pp. 95-124). Buenos Aires: Grupo de Zooarqueología de Camélidos.

Olivera, D. E. y Grant, J. (2009). Puestos de altura de la Puna argentina: zooarqueología de Real Grande 1 y 6 y Alero Tomayoc. Revista del Museo de Antropología, 2, 151-168.

Passey, B., Robinson, T., Ayliffe, L., Cerling, T., Sponheimer, M., Dearing, M., Roeder, B. L. y Ehleringer, J. (2005). Carbon isotope fractionation between diet, breath $\mathrm{CO}_{2}$, and bioapatite in different mammals. Journal of Archaeological Science, 32, 1459-1470.

Post, D. (2002). Using stable isotopes to estimate trophic position: models, methods, and assumptions. Eco$\log y, 83(3), 703-718$

Samec, C. T. (2014). Ecología isotópica en la Puna Seca Argentina: un marco de referencia para el estudio de las estrategias de pastoreo en el pasado. Cuadernos del Instituto Nacional de Antropología y Pensamiento Latinoamericano, Series Especiales, 2(1), 61-85.

Samec, C., Morales, M., Pirola, M., Panarello, H. y Yacobaccio, H. (2017). Composición isotópica del oxígeno e hidrógeno en aguas meteóricas y superficiales de la Provincia de Jujuy, Argentina. Trabajo presentado en el Segundo Taller Arqueología e Isótopos Estables en el Sur de Sudamérica. San Rafael, Argentina.

Samec, C. T., Morales, M. R. y Yacobaccio, H. D. (2014). Exploring human subsistence strategies and environmental change through stable isotopes in the Dry Puna of Argentina. International Journal of Osteoarchaeology, 24(2), 134-148.

Samec, C. T., Pirola, M., Yacobaccio, H. D. y Panarello, H. O. (2020). Assessing prehispanic herding strategies through stable isotope analysis: A case study from the Dry Puna of Argentina. Environmental Archaeology, 25(3), 353-364.

Samec, C. T., Yacobaccio, H. D. y Panarello, H. O. (2017). Carbon and nitrogen isotope composition of natural pastures in the Dry Puna of Argentina: A baseline for the study of prehistoric herd management strategies. Archaeological and Anthropological Sciences, 9(2), 153-163.

Samec, C. T., Yacobaccio, H. D. y Panarello, H. O. (2018). Stable isotope compositions of South American camelids in the Dry Puna of Argentina: A frame of reference for the study of prehistoric herding and hunting strategies. Journal of Archaeological Science: Reports, 18, 628-636. 
Schutkowski, H. (2006). Human Ecology: Biocultural Adaptations in Human Communities. Berlin-Heidelberg: Springer.

Solá, P. (2007). La cerámica utilitaria de grupos pastoriles en Susques (Puna argentina). En Cremonte, M. B. y Ratto, N. (Eds.). Cerámicas arqueológicas. Perspectivas arqueométricas para su análisis e interpretación (pp. 73-95). CD ROM. San Salvador de Jujuy: EdUNJu.

Solá, P. (2011). Tiestos trazadores de cerámica exótica en sitios tardíos de los alrededores de Susques (Jujuy, Puna argentina). Intersecciones en Antropología, 12, 177-194.

Srur, M. G., Izeta, A. D. y Scattolin, M. C. (2012). Alimentación de camélidos sudamericanos en los sitios formativos de Cardonal y Bordo Marcial (Catamarca, Argentina): Una aproximación isotópica. Archaeobios, 6(1), 5-18.

Stuiver, M. y Reimer, P. J. (1993). Extended ${ }^{14} \mathrm{C}$ data base and revised CALIB $3.0{ }^{14} \mathrm{C}$ age calibration program. Radiocarbon, 35(1), 215-230.

Szpak, P., Millaire, J. F., White, C. D. y Longstaffe, F. J. (2014). Small scale camelid husbandry on the north coast of Perú (Virú Valley): insight from stable isotope analysis. Journal of Anthropological Archaeology, 36, $110-29$.

Tykot, R. H. (2004). Stable Isotopes and Diet: You Are What You Eat. En M. Martini, M. Milazzo y M. Piacentini (Eds.) Physics Methods in Archaeometry. Proceedings of the International School of Physics "Enrico Fermi” Course CLIV (pp. 433-444). Bologna: Società Italiana di Fisica.

Yacobaccio, H. D. (2007). Andean camelid herding in the South Andes: ethoarchaeological models for archaeozoological research. Anthropozoologica, 42(2), 143-154.

Yacobaccio, H. D., Catá, M. P., Morales, M. R., Solá, P., Alonso, M. S., Rosenbusch, M., Vázquez, C., Samec, C. T., Oxman, B. I. y Cáceres, M. (2011). El uso de cuevas por pastores andinos: el caso de Cueva Quispe (Susques, Puna de Jujuy). En Muscio, H. y López, G. (Eds.). Arqueología en las Tierras Altas de Argentina. Evolución y Cambio Cultural (pp. 33-47). British Archaeological Reports (BAR). Oxford: Editorial John Hedges.

Yacobaccio, H. D., Madero, C. M. y Malmierca, M. P. (1998). Etnoarqueología de Pastores Surandinos. Grupo Zooarqueología de camélidos. Buenos Aires.

Yacobaccio, H. D., Madero, C. M., Malmierca, M. P. y Reigadas, M. C. (1997-1998). Caza, domesticación y pastoreo de camélidos en la Puna Argentina. Relaciones de la Sociedad Argentina de Antropología, XXIIXXIII, 389-418.

Yacobaccio, H. D., Morales, M. R. y Samec, C. T. (2009). Towards an isotopic ecology of herbivory in the Puna ecosystem: New results and patterns on Lama glama. International Journal of Osteoarchaeology, 19(2), $144-155$.

Yacobaccio, H. D. y Vilá, B. (2016). A model for llama (Lama glama Linnaeus, 1758) domestication in the southern Andes. Anthropozoologica, 51(1), 5-13.

Zhou, J. y Lau, K. M. (1998). Does a monsoon climate exist over South America? Journal of Climate, 11, 1020-1040. 